\title{
Analysis of ocean internal waves imaged by multichannel reflection seismics, using ensemble empirical mode decomposition
}

\author{
Haibin Song ${ }^{1}$, Yang Bai ${ }^{1,2}$, Luis Pinheiro ${ }^{3}$, Chongzhi Dong ${ }^{4}$, \\ Xinghui Huang ${ }^{1,2}$ and Boran Liu ${ }^{1,2}$ \\ ${ }^{1}$ Key Laboratory of Petroleum Resources Research, Institute of Geology and Geophysics, \\ Chinese Academy of Sciences, Beijing 100029, People's Republic of China \\ ${ }^{2}$ Graduate University of Chinese Academy of Sciences, Beijing 100049, People's Republic of China \\ ${ }^{3}$ Departamento de Geociências and CESAM, Universidade de Aveiro, 3800 Aveiro, Portugal \\ ${ }^{4}$ Key Laboratory of Submarine Geosciences, Second Institute of Oceanography, Hangzhou 310012, \\ People's Republic of China \\ E-mail: baiyang@mail.iggcas.ac.cn
}

Received 1 September 2011

Accepted for publication 3 April 2012

Published 26 April 2012

Online at stacks.iop.org/JGE/9/302

\begin{abstract}
Research on ocean internal waves using seismic oceanography is a frontier issue both for marine geophysicists and physical oceanographers. Images of the ocean water layer obtained by conventional processing of multichannel seismic reflection data can show the overall patterns of internal waves. However, in order to extract more information from the seismic data, new tools need to be developed. Here, we use the ensemble empirical mode decomposition (EEMD) method to decompose vertical displacement data from seismic sections and apply this method to a seismic section from the northeastern South China Sea, where clear internal waves are observed. Compared with the conventional empirical mode decomposition method, EEMD has greatly reduced the scale mixing problems induced in the decomposition results. The results obtained show that the internal waves in this area are composed of different characteristic wavelengths at different depths. The depth range of 200-1050 m contains internal waves with a wavelength of $1.25 \mathrm{~km}$ that are very well coupled in the vertical direction. The internal waves with a wavelength of $3 \mathrm{~km}$, in the depth range of 200-600 m, are also well coupled, but in an oblique direction; this suggests that the propagation speed of internal waves of this scale changes with depth in this area. Finally, the internal waves with a wavelength of $6.5 \mathrm{~km}$, observed in the depth range of 200-800 m, are separated into two parts with a phase difference of about $90^{\circ}$, by a clear interface at a depth of $650 \mathrm{~m}$; this allows us to infer an oblique propagation of wave energy of this scale.
\end{abstract}

Keywords: seismic oceanography, ocean internal waves, EMD, EEMD

(Some figures may appear in colour only in the online journal)

\section{Introduction}

Ocean internal waves are waves that oscillate within, rather than at the surface of seawater when there is density stratification. The frequency of ocean internal waves varies between the inertial frequency and the Brunt-Vaisala frequency. Ocean internal waves are the most important part of the ocean energy cascade. The generation, propagation and dissipation of ocean internal waves cause great energy exchange with an important influence on the ocean dynamic processes. Unlike surface waves, the amplitude of internal waves can be very large, sometimes greater than $100 \mathrm{~m}$ (Liu 
et al 1998); therefore they can have major impacts on the safety of marine engineering, ocean communications, oil exploration (Cai and Gan 2001) or marine military facilities (Jiang et al 2009). Therefore, the research on ocean internal waves is of great concern to a large number of scientists from various countries.

The development of research on ocean internal waves is however highly dependent on the quality and quantity of observational data. Since the early 1970s, with the rapid development of oceanography, the intense development of new instrumentation tools to probe the oceans allowed great progress to be made in research on ocean internal waves. Garrett and Munk (1972) proposed the GM72 model spectra for ocean internal waves, and through constant improvement, new editions of this model have been proposed subsequently (Garrett and Munk 1975, 1979), which are also referred to as GM model spectra. Although these spectra are essentially statistical descriptions of oceanic phenomena, they have been a key reference in analysing the data from ocean internal waves. One of the limitations of conventional physical oceanography instrumentation, such as XBTs (expendable bathythermographs), CTDs (conductivity, temperature and depth sensors), XCTDs (expendable CTDs) and ADCPs (acoustic Doppler current meters), is that they cannot provide vertical sections of the ocean in a very short time and lack good lateral resolution. Remote sensing cannot also provide the necessary subsurface water information directly (Fang and Du 2005).

In 2003, Holbrook showed that there were weak reflection signals inside the water that could be correlated with the ocean structure (Holbrook et al 2003); this gave rise to an exciting new research field: seismic oceanography. Holbrook and Fer (2005) further noted that there were continuous undulations in the reflection section of seawater. They compared the spectra of these undulations with the GM76 model spectra, and proposed that these undulations were representations of ocean internal waves, hence laying the foundations for the research on ocean internal waves using the multichannel seismic reflection method. Compared with conventional oceanography measurements, seismic reflection can provide vertical sections of the water layer in a very short time, with the required high lateral resolution to image internal waves.

The northeastern South China Sea is an excellent internationally recognized natural laboratory for these types of studies (Fang and Du 2005). Song et al (2009) processed a seismic section from the northeast South China Sea and showed that the undulations of the reflections in the stacked seismic section exhibited the overall patterns of internal waves. Dong et al (2009) computed the wavenumber spectra ${ }^{5}$ of these undulations and found that the overall patterns of those spectra were very similar to the GM76 model spectra. More recently, Song et al (2010) decomposed part of that section using the EMD (empirical mode decomposition) method Huang et al (1998), with encouraging results. However, recent studies showed that, although useful, the EMD method has some

\footnotetext{
5 Since the signals analysed here are a function of distance (space) and not time, therefore instead of frequency spectrum we will always use the term wavenumber spectrum.
}

severe drawbacks, such as scale mixing problems (Huang et al 1999), which cause some difficulties in the physical analysis of the results. In order to overcome the drawbacks of EMD, Wu and Huang (2009) more recently proposed an improved method called ensemble EMD (EEMD). This paper investigates the characteristics of ocean internal waves in northeastern South China Sea using EEMD.

\section{The EMD method and its drawbacks}

Unlike almost all previous signal analysis methods, the EMD method is adaptive. Huang et al (1999) have shown that all signals can be interpreted as composed of a series of IMFs (intrinsic mode functions) and the objective of EMD is precisely to decompose signals into series of IMFs and a residual (1):

$$
\mathbf{X}(t)=\sum_{i=1}^{n} \mathbf{c}_{i}+\mathbf{r}_{n},
$$

where $\mathbf{X}(t)$ is the signal to be decomposed, $\mathbf{c}_{i}$ are the resultant IMFs and $\mathbf{r}_{n}$ are residuals.

IMFs are oscillatory functions with varying amplitude and frequency, which are defined as follows: (1) throughout the whole length of an IMF set, the number of extrema (including all maximum and minimum) and the number of zero-crossings must either be equal or differ at most by $1 ;(2)$ at any data location, the mean value of the envelope defined by the local maxima and the envelope defined by the local minima is zero (actually, it is not strictly zero but controlled by a certain stop criterion).

In practice, the IMFs are obtained through a sifting process.

(1) Firstly, all the local maxima are identified and connected with cubic splines to obtain the upper envelope of the signal (the length of the envelope should be the same as the original data set); this process is then repeated for the local minima to obtain the lower envelope of the signal (the whole data set variation should then be within the range between the two envelopes).

(2) Secondly, the mean of the upper and lower envelopes $\left(\mathbf{m}_{1}\right)$ is calculated and the difference $\left(\mathbf{h}_{1}\right)$ between the original data and $\mathbf{m}_{1}$ determined:

$$
\mathbf{X}(t)-\mathbf{m}_{1}=\mathbf{h}_{1} .
$$

This process is known as the 'one sifting process'; ideally, $\mathbf{h}_{1}$ should be an IMF, but this is not usually the case. Consequently, the sifting process should be repeated several times until the results satisfy the definitions of IMFs

$$
\begin{gathered}
\mathbf{h}_{1}-\mathbf{m}_{11}=\mathbf{h}_{11}, \quad \mathbf{h}_{11}-\mathbf{m}_{12}=\mathbf{h}_{12} \ldots \\
\mathbf{h}_{1(k-1)}-\mathbf{m}_{1 k}=\mathbf{h}_{1 k} .
\end{gathered}
$$

(3) Then, denoting the final result $\left(\mathbf{h}_{1 k}\right)$ by $\mathbf{c}_{1}$ and the residual $\left(\mathbf{X}(t)-\mathbf{c}_{1}\right)$ by $\mathbf{r}_{1}, \mathbf{c}_{1}$ is the first component while $\mathbf{r}_{1}$ is now treated as the data and the decomposition process is repeated again and again so as to obtain $\mathbf{c}_{2}, \mathbf{c}_{3}, \ldots, \mathbf{c}_{n}$ in turn, and a residual $\mathbf{r}_{n}$.

In terms of its practical application, the EMD method mainly has the following two main drawbacks: (1) sometimes, there will be scale mixing problems in some IMFs; (2) the EMD is too sensitive to some local tiny changes. 


\section{The EEMD method}

In order to overcome some of the drawbacks of EMD, Wu and Huang (2009) proposed an improved method, called EEMD. Based on EMD, the EEMD method improves the results of the decomposition through the following two techniques: (1) noise-assisted data analysis, i.e. the addition of finite amplitude (usually small) white noise to the data before decomposition; (2) the application of a simple statistics principle-stacking the white noise many times will suppress that noise.

If the wavenumber spectra are continuous (i.e. if they have a continuous representation of all constituting components of different scales), the centre wavenumber of the spectra of the successive IMFs, obtained through the EMD sifting process, will be approximately halved (Wu and Huang 2004, Flandrin et al 2004). However, when the wavenumber spectrum is not continuous, although the centre wavenumber for each successive IMF spectra will still decrease, there are no fixed rules in this case, and this often causes scale mixing problems in the data analysis, especially when processing $2 \mathrm{D}$ data sets (such as images or seismic sections). For 2D data sets, such as the one analysed here, that result from the picking of the main reflectors in the horizontal direction from a seismic section (see figure 1), these are treated as composed of a vertical stack of 1D data sets, each of which is decomposed in the horizontal direction.

What often happens is that the information from similar scales is distributed in different levels of IMFs (i.e. the information concerning a certain scale can be distributed between the second and third level IMFs) and/or IMFs of a certain level contain information from various different scales. However, when we work with images and combine the IMFs, we usually combine the IMFs of the same level as one component (of the image). For example, we combine all the IMFs of the second level as one component, and all the IMFs of the third level as another component. If in one of the components there is information from different scales, then there is scale mixing. Sometimes, scale mixing problems can be avoided by adjusting some IMFs manually; however, when the data volume is too large, this will be inefficient and, moreover, when a single IMF has scale mixing problems it is meaningless to do any adjustment.

However, when we apply the EEMD method ( $\mathrm{Wu}$ and Huang 2009), we will first add finite amplitude white noise to the data (with the same length as the data set). This will make the wavenumber spectrum energy (maybe not continuous originally) more continuous, because the added energy of the white noise will be evenly distributed throughout the wavenumber spectrum. In such a case, the ranges of wavenumber spectra of the successive IMFs will again decrease by a factor of about 2 (Wu and Huang 2009). This will then reduce the scale mixing problems.

Although we can reduce the scale mixing problems by adding white noise to the data, the result of the decompositions may not look good because of the added noise; however, we can use the stacking technique, mentioned above, to reduce or cancel significantly the added noise. We repeat the noiseadded decompositions a certain number of times (called the ensemble number-NE), with different white noise added each time; then we stack and average the IMFs of the same level, to suppress the added white noise (since the addition of many white noise sequences will statistically tend to cancel each other). Theoretically, if the ensemble number approaches infinity, the white noise will be fully suppressed to zero. However, in practice, stacking for about 100 times is generally good enough. It should be noted that, because of the added noise, sometimes there are IMFs containing no information on the original data since they purely consist of noise; these IMFs will generally approach zero after stacking.

In summary, the implementation steps for EEMD are as follows (Wu and Huang 2009).

(1) Add white noise to the data and apply EMD.

(2) Subtract the same white noise used by step (1) from the data, and apply EMD.

(3) Stack the results of steps (1) and (2).

(4) Repeat steps (1)-(3) several times (NE times) with different white noise added each time.

(5) Stack all the IMFs of the same level and divide the result by $2 \times \mathrm{NE}$, to obtain the average.

The purpose of steps (1) and (2) is to suppress the white noise more efficiently with a certain finite number of stacks. Some points should nevertheless be noted: (1) sometimes IMFs of different levels should be combined to obtain a result with more physical meaning (see the discussion above); (2) frequently, it is also not necessary to decompose the data until it cannot be decomposed any further; we can decompose as we need, and treat the remaining as a residual; however, it should be noted that the number of IMFs should not exceed $\left\lfloor\log _{2}(N)\right.$ $-1\rfloor$, where $N$ is the length of data, i.e. the number of samples, and \lfloor\rfloor denotes the floor function ${ }^{6}$ of the expression inside; (3) theoretically, we can suppress any finite amplitude white noise by performing an infinite number of stacks; however, since the ensemble number is finite and we aim to highlight small undulations in the data, it is better to add white noise with only a small energy.

Appendix A and appendix B give some detailed discussions and illustrations of how EEMD can solve the two main drawbacks that EMD has.

\section{Application of EEMD to the analysis of ocean internal waves}

Song et al (2010) processed a seismic section (figure 1) from the northeastern South China Sea, and got the vertical displacement distribution of internal waves shown in figure 2. These authors then decomposed figure 2 in the common mid point (CMP) direction (horizontal), using the EMD method; from the resulting low wavenumber components they were able to image the fine structure of the internal waves, as shown in figure 3, and get new insights into their structure and kinematics. Figure 3(a) shows 10-11 positive and negative vertical displacement bands, well coupled in the vertical direction in

6 The floor function of $x$, also called the greatest integer function or integer value, gives the largest integer less than or equal to $x$. 


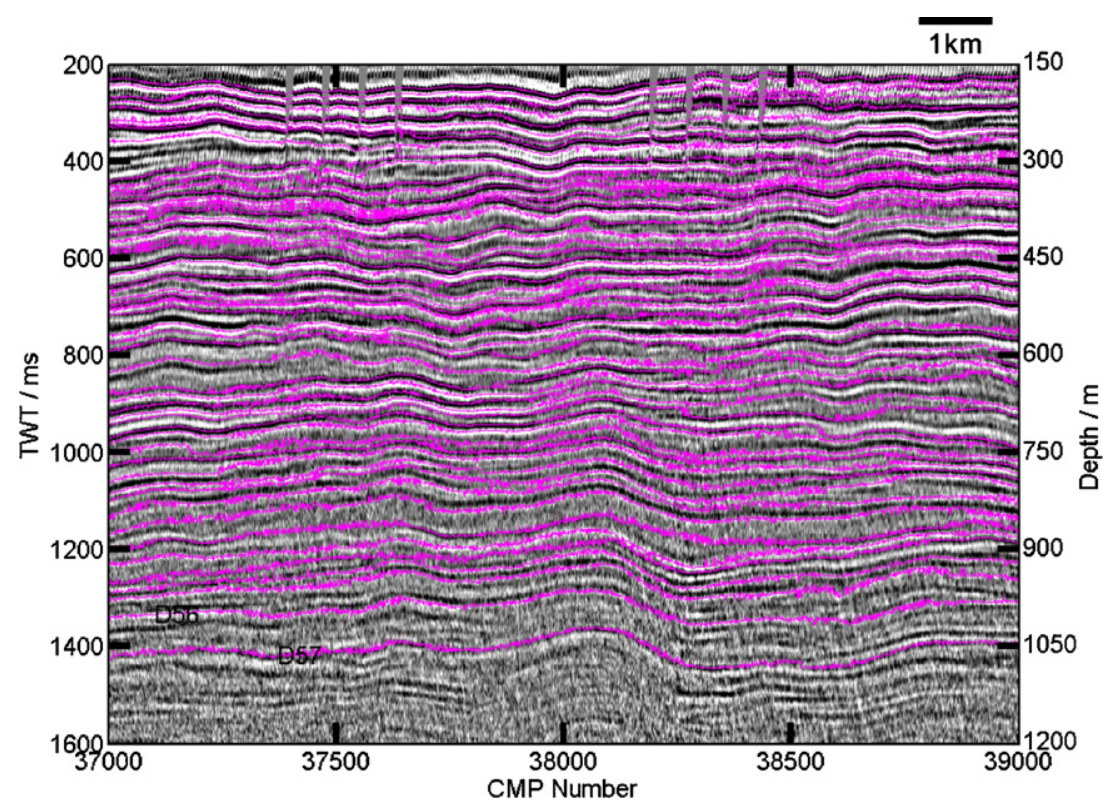

Figure 1. Seismic reflection section of the water column from the northeastern South China Sea (Song et al 2010). The purple lines denote the picked horizons (such as D56 and D57). TWT is the two-way travel time; CMP stands for common mid point.

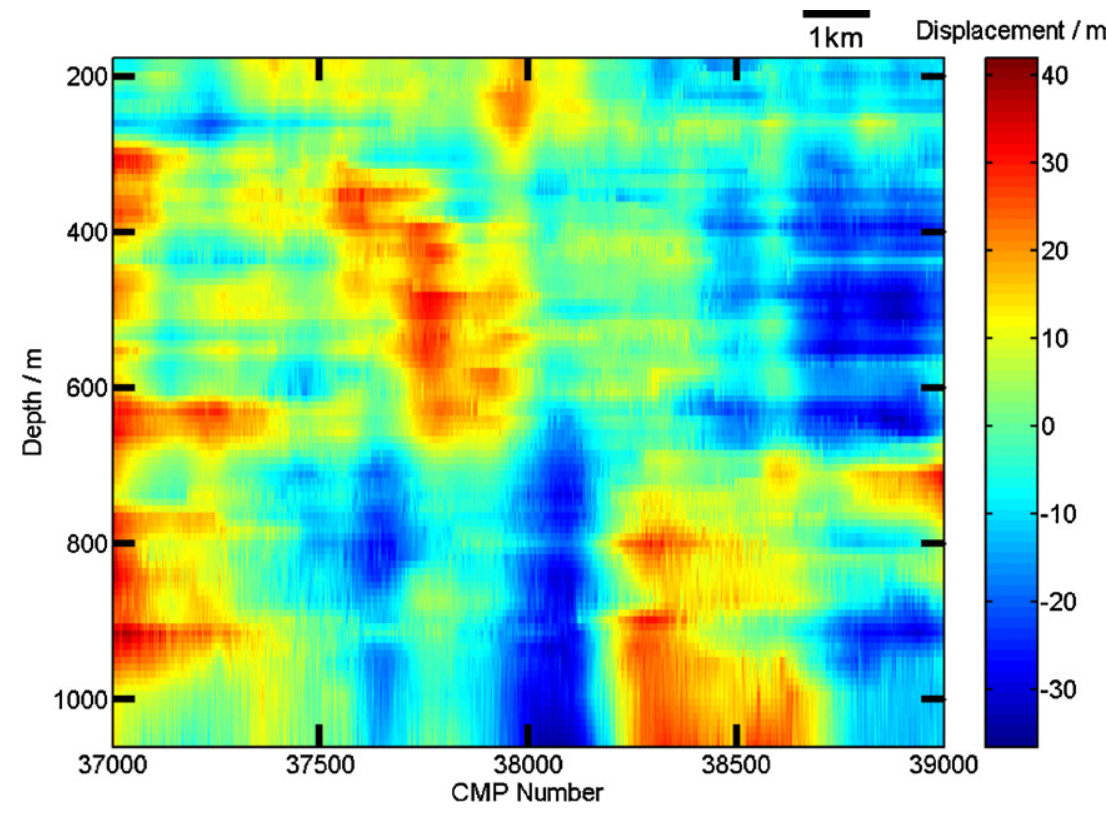

Figure 2. The vertical displacement distribution of internal waves computed from figure 1, by subtracting the trend from each picked horizon in the original data.

the depth range of 200-900 $\mathrm{m}$. Figure 3(b) shows 5-6 positive and negative vertical displacement bands, well coupled in the vertical direction in the depth range of $600-1000 \mathrm{~m}$. The waves shown in figures $3(c)$ and $(d)$, however, show a large difference between the upper and lower areas, separated by clear interfaces at 600 and $700 \mathrm{~m}$, respectively. In particular for figure $3(d)$, the phase difference is about $90^{\circ}$, which was interpreted as evidence of oblique propagation of the wave energy.

A more careful analysis, however, shows that the four components obtained with EMD have evidence of scale mixing. In figure $3(a)$ the scales of areas (1) and (2) are similar to the overall scale of figure $3(b)$. In figure $3(b)$, the scales of area (4), denoted by four rectangles, are also similar to the overall scale of figure $3(a)$, and the pattern of area (3) is almost the same as that of area (6) in figure $3(c)$. As such, it appears better to incorporate area (5) in figure $3(b)$ into the corresponding place in figure $3(c)$, and to incorporate area (7) in figure 3(c) into the corresponding place in figure 3(d). This brief analysis shows that figure 3(b) clearly has various scale mixing problems, almost everywhere. Also, there are many 'stratified' patterns in the four components, such as the lower part of area (3), in figure 3(b). This is also a type of scale mixing, and the missing part of the 'stratified' patterns can always be found in the neighbouring components. The existence of these scale mixing problems has greatly affected 

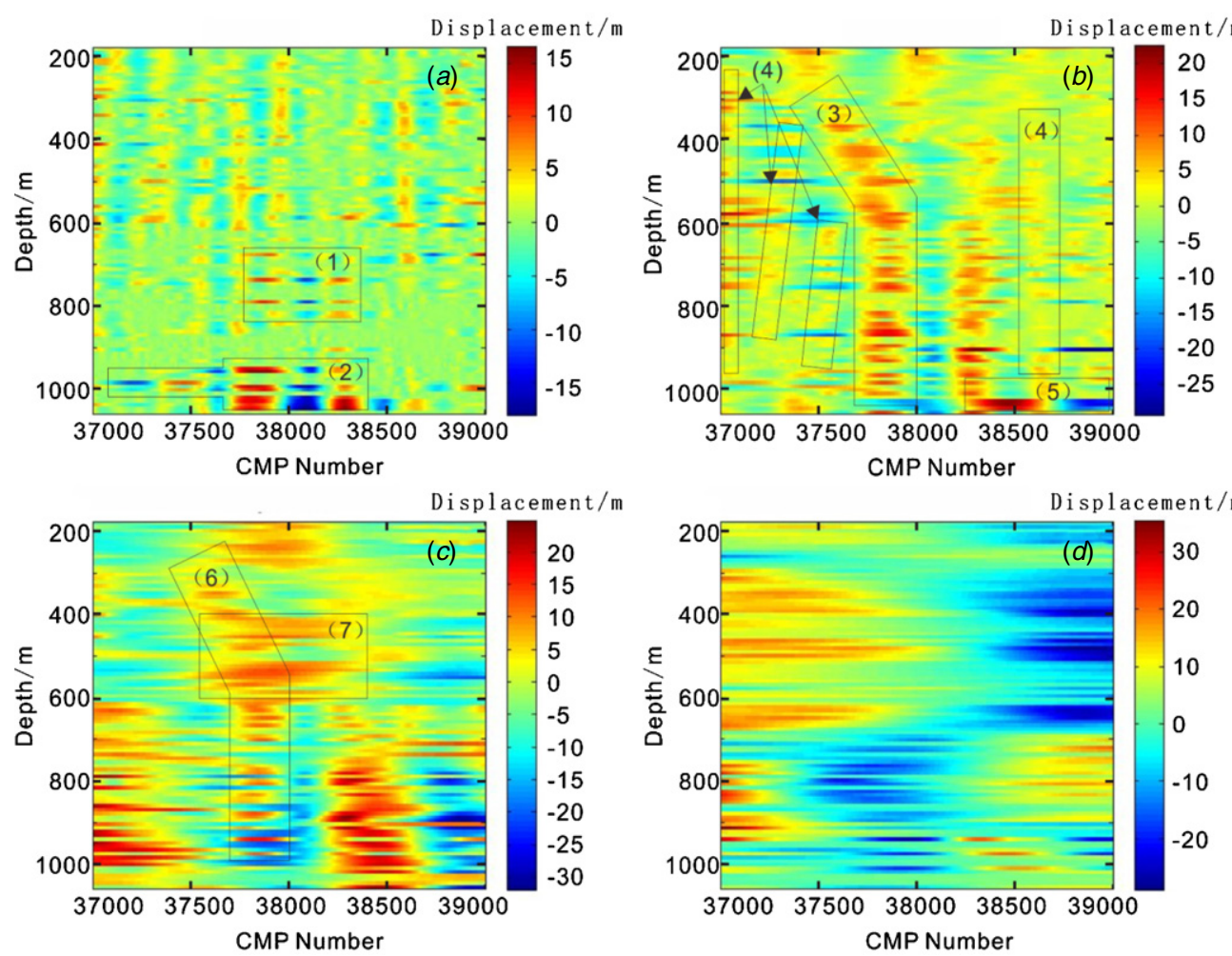

Figure 3. The four low wavenumber components of the EMD decomposition of figure 2: (a) shows 10-11 positive and negative vertical displacement bands well coupled in the vertical direction, while $(b)$ shows 5-6; $(c)$ and $(d)$ show a large difference between the upper and lower areas, separated by clear interfaces at 600 and $700 \mathrm{~m}$, respectively. Areas marked by rectangles are those with various kinds of scale mixing problems: the scales of areas denoted by (1), (2), (5) and (7) are obviously different from their background scales; area (3) and area (6) almost show the same pattern; the scale of area (4) in $(b)$ is more similar to the overall scale of $(a)$. The 'stratified' patterns in all these four components are also the results of scale mixing.
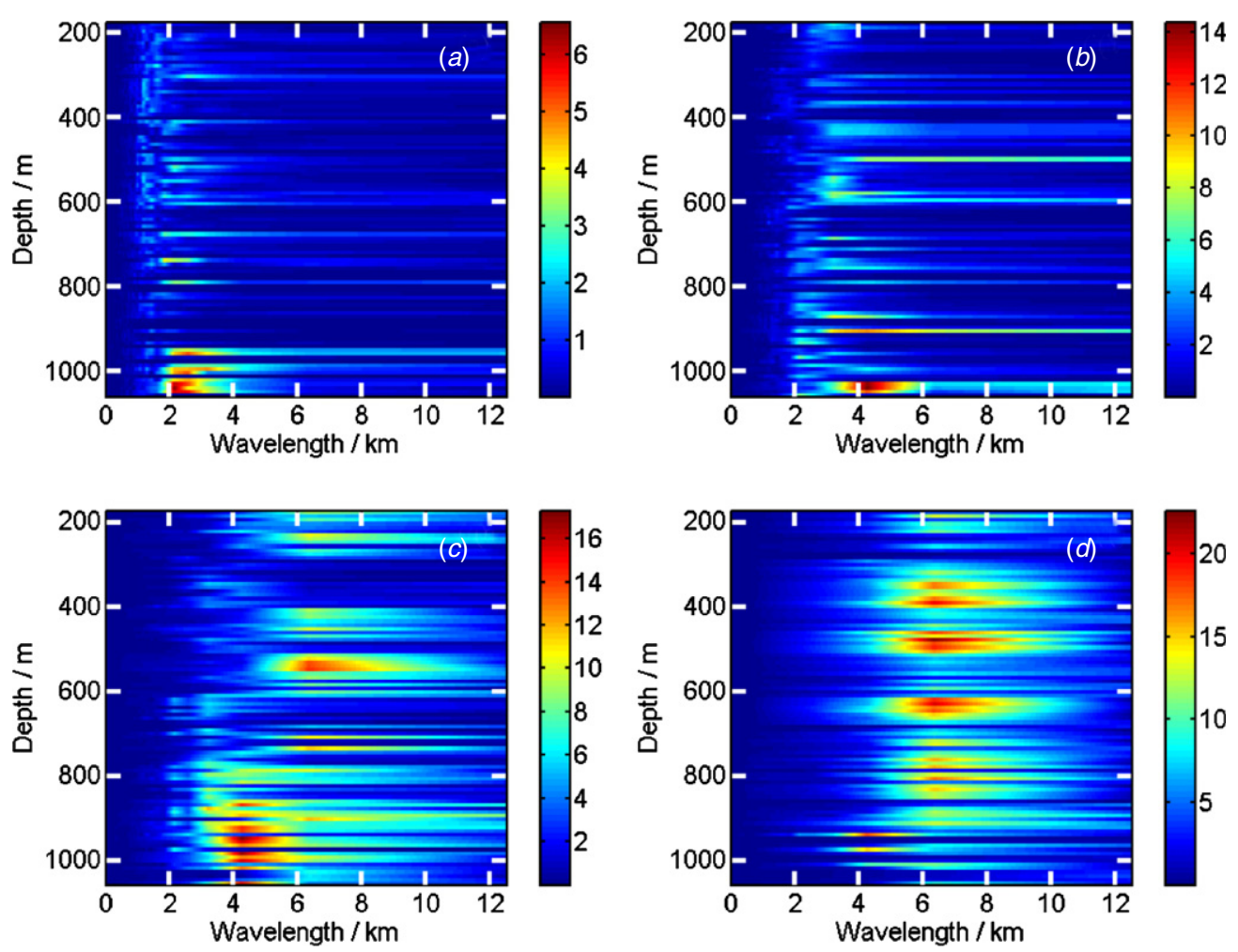

Figure 4. The corresponding wavenumber spectra of figure 3. Affected by the scale mixing problems, all four wavenumber spectra show a 'stratified' pattern, and the wavelength range with significant energy is hard to estimate precisely. 

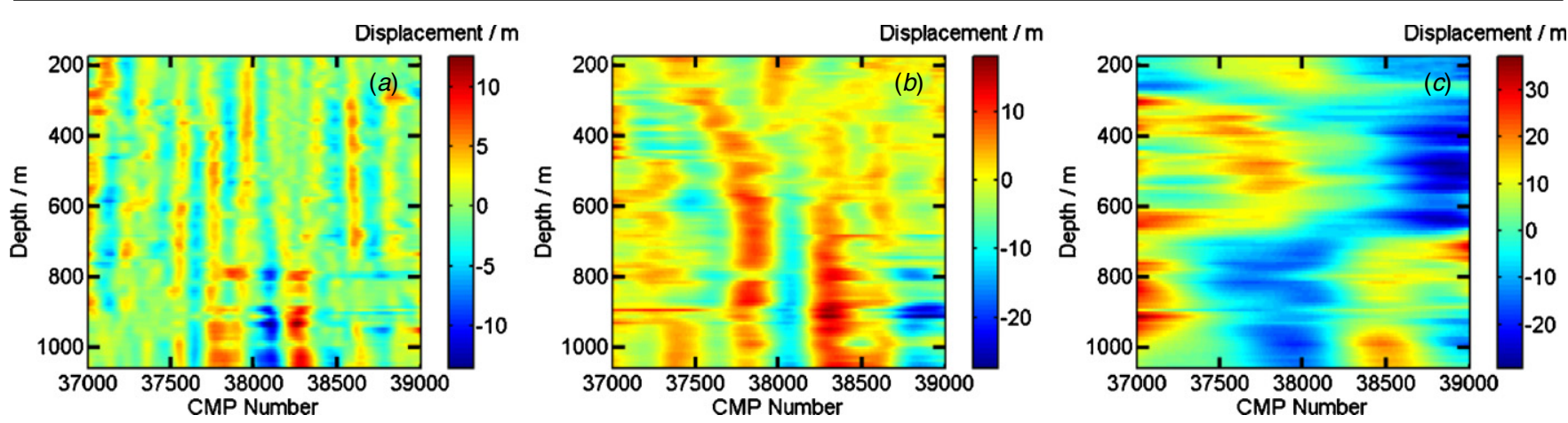

Figure 5. The three low wavenumber components of the EEMD decomposition of figure 2. Compared with figure 3, it is clear that the scale mixing problems are greatly reduced, although some are still present, such as in the middle lower part of $(a)$ and left lower part of $(b)$. The structures shown here are much clearer than those of figure 3.
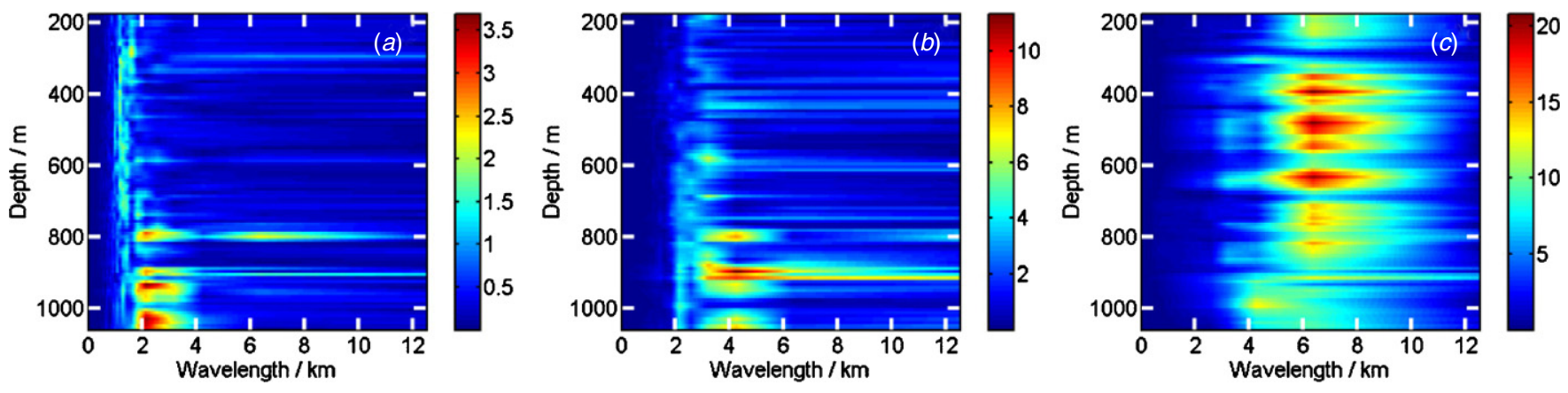

Figure 6. The corresponding wavenumber spectra of figure 5. Due to the reduction of scale mixing problems, the wavelength range for each single component is now much more concentrated than before, and more continuous in the vertical direction. Despite the remaining scale mixing problems in the lower part of $(a)$ and $(b)$, it is still easy to estimate that the characteristic wavelength for $(a)$ is about $1.25 \mathrm{~km}$; for $(b)$, it is about $3 \mathrm{~km}$ for the upper part and $2.2 \mathrm{~km}$ for the lower part; for $(c)$ it is about $6.5 \mathrm{~km}$.

the previous interpretation of the physical meaning of the patterns represented by the four components, especially the estimation of the wavenumber range, and then the estimation of wavelength range (figure 4).

Figure 5 shows the decompositions of figure 2, now using the EEMD method (with the ensemble number NE $=100$ ). Here, it is clear that the main components become three rather than the original four. Compared with figure 3 , it is clear that the scale mixing problems were greatly reduced, although some are still present, such as in the middle lower part of figure 5(a) and left lower part of figure 5(b). With the scale mixing problems greatly reduced, the 'stratified' patterns almost disappeared, revealing a much clearer image of the fine structure of the ocean internal waves. Figure 6 shows the wavenumber spectra corresponding to the three components of figure 5. Comparing figure 6 with figure 4 , due to the reduction of the scale mixing problems, the wavelength range for each single component is now much more concentrated than before, and more continuous in the vertical direction. This provides a much more reliable basis to estimate the wavelength range of each component. From the analysis of the wavenumber spectra, the observed pattern in figure 5(a) is mainly composed of internal waves with a wavelength of $1.25 \mathrm{~km}$, which couple well in the depth range of 200-1050 m. Because there are still some scale mixing problems in the middle lower part, other energy peaks with a wavelength of $2-3 \mathrm{~km}$ in figure $6(a)$, at a depth of about $800 \mathrm{~m}$, and also in the depth range of 900-1000 m, are nevertheless observed. The upper part above
$600 \mathrm{~m}$ in figure $5(b)$ mainly consists of internal waves with a wavelength of $3 \mathrm{~km}$, and the waves in the middle upper part couple well in an oblique direction, with the apparent angle of about $30^{\circ}$ to the vertical direction; from this observation, we infer that the internal waves of this scale, in this part of the section, have different propagating speed with depth. The lower part below $600 \mathrm{~m}$ mainly consists of internal waves with a wavelength of $2.2 \mathrm{~km}$, well coupled in the vertical direction. Like the situation in figure 6(a), there are other energy peaks around $4 \mathrm{~km}$ wavelength in the lower part of the section, at a depth of about $800-900 \mathrm{~m}$, and beneath $1000 \mathrm{~m}$ in figure $6(b)$, in addition to the peaks around the $2.2 \mathrm{~km}$ wavelength. The upper part above $850 \mathrm{~m}$ in figure 5(c) mainly consists of internal waves of $6.5 \mathrm{~km}$ wavelength, while the lower part mainly consists of internal waves of $5 \mathrm{~km}$ wavelength. However, because of the influence of scale mixing, the energy of the lower part in figure $6(c)$ is relatively small. With an interface at $650 \mathrm{~m}$, the upper and lower parts of figure $5(\mathrm{c})$ have a phase difference of about $90^{\circ}$, which can be interpreted as indicative of the oblique propagation of wave energy at this scale.

\section{Conclusions}

The seismic section of the water column obtained from the processing of one multichannel seismic reflection line from the northeastern South China Sea shows undulations in the 
reflectors that likely correspond to the stacking of internal waves of various scales, and therefore can only show their overall pattern. The previous application of the EMD method to this seismic section allowed the extraction of the fine structure of the internal waves, at various scales. However useful the EMD proved to be for this purpose in a first approach, some drawbacks of this method nevertheless remained unsolved, such as scale mixing and sensitivity, which affected the decomposition of the data. As such, in this paper, we applied an advanced version of EMD, called EEMD, to reprocess the same section from the northeastern South China Sea. Comparing the new decompositions with the results previously obtained with EMD we could largely solve the previous scale mixing problems, which are now greatly reduced. The upper part of the section, above a water depth of about $800 \mathrm{~m}$, rarely contains any scale mixing problems. The lower part, however, around the depth of about $800 \mathrm{~m}$ and in the range of 900-1050 $\mathrm{m}$ still has a few remaining scale mixing problems. The resulting wavenumber spectra of the various components now show the energy much more concentrated in the wavenumber range than before, and more continuous in the vertical direction, which provides a much more sound basis to better estimate the wavelength range of each of the components.

The new results show that there are internal waves of $1.25 \mathrm{~km}$ wavelength in the depth range of 200-1050 m, very well coupled in the vertical direction. Also, internal waves of $3 \mathrm{~km}$ wavelength were observed in the depth range of 200$600 \mathrm{~m}$, and part of this scale also is well coupled in an oblique direction, from which we infer that internal waves of this scale in this part of the section have different propagating speed with depth. In the depth range of 600-1050 m, internal waves of $2.2 \mathrm{~km}$ wavelength, well coupled in the vertical direction, were also imaged. Finally, above $850 \mathrm{~m}$ water depth, there are internal waves of $6.5 \mathrm{~km}$ wavelength, separated by a clear interface at a depth of about $650 \mathrm{~m}$, which show a clear phase difference of about $90^{\circ}$ between the upper and lower parts; this is interpreted as indicating an oblique propagation of wave energy at this scale.

\section{Acknowledgments}

This research was supported co-financially by the National Major Fundamental Research and Development Project of China (no 2011CB403503) and China NSF (nos 91028002, 41076024). We would like to thank Professor Barry Ruddick for help during our seismic oceanographic cooperation. We are grateful to Dr Zhaohua Wu and Professor Norden Huang for their kind permission to use their modelling data. Our thanks are also given to the anonymous reviewers for very helpful suggestions and detailed comments.

\section{Appendix A. Scale mixing problems}

Scale mixing problems in 1D signals are often caused by intermittency (Wu and Huang 2009, Wu et al 2009). Not only the intermittency of the whole signal, but also intermittency at certain scales will lead to scale mixing. There are three main types of causes for scale mixing.
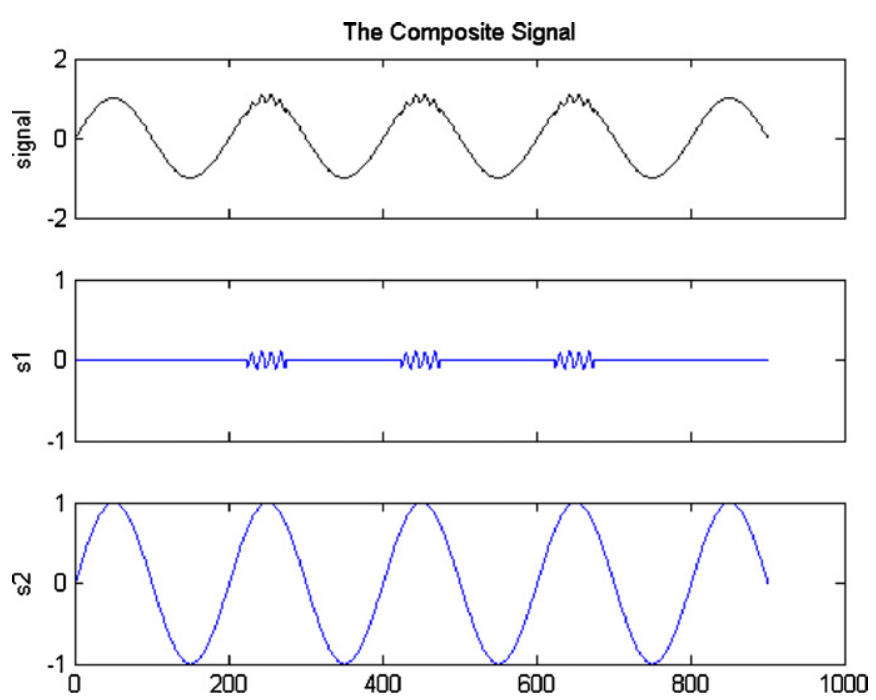

Figure A1. The signal used for testing the method is composed of the sum of two signals: s1 and s2 (after Huang et al (1999)). s1 is intermittent and its scale is comparatively small. $\mathrm{s} 2$ is a simple sinusoidal signal with a much larger scale. The vertical axis represents the amplitude of the signal and the horizontal axis represents the sampling points, without any particular physical meaning.

(1) Information from different scales exists in a single IMF.

(2) Information from the same scale is distributed between different levels of IMFs.

(3) When there are some relatively large (or small) values in a certain IMF, often a neighbouring IMF will have relatively small (or large) values at the corresponding position. This type of feature is artificial and has no physical meaning.

Tackling scale mixing problems is therefore extremely important because they will cause severe difficulties in the interpretation of the physical processes responsible for the generation of the data analysed.

Below, we show an example from Huang et al (1999) and $\mathrm{Wu}$ and Huang (2009), which illustrates how scale mixing problems occur when EMD is applied, and how they can be solved by EEMD. As mentioned before, scale mixing problems occur mainly because of intermittency in the signal. This makes the distribution of extrema non-uniform and therefore the range defined by the upper and lower envelopes, interpolated from the extrema, cannot cover the data completely and the mean of the upper and lower envelopes does not represent the exact trend of the signal (see, for example, figure A2 $(a)$, middle image). It should also be noted that, each time the sifting process subtracts the estimated trend from the signal, it will also likely subtract information concerning other scales from the original signal. Therefore, if the signal trend cannot be correctly defined, the final result, which will be an IMF, will of course have information from other scales and scale mixing problems will emerge. However, the added white noise will make the extrema be distributed uniformly throughout the whole signal when applying EEMD, and therefore the envelopes and trend can be defined more accurately, thereby reducing the scale mixing problems.

Figure A1 shows the signal used in this example, which is composed of the sum of two signals: s1 and s2. $\mathrm{s} 1$ is 

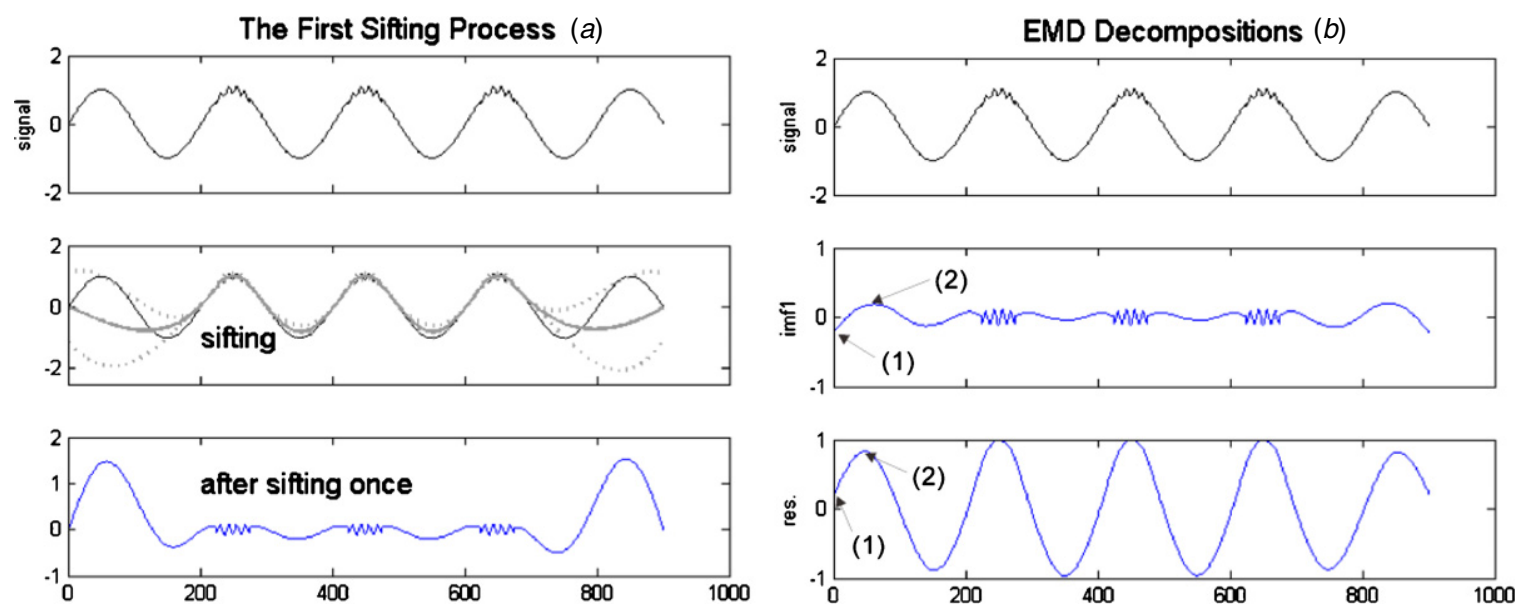

Figure A2. (a) The first sifting process and the corresponding result (recalculated after Wu and Huang (2009)). The first image is the original signal. The second image shows the process of sifting, in which the black solid line is the signal, the grey dotted lines are the envelopes (upper and lower) and the grey solid line is the mean of the envelopes. The third image is the result after sifting once. (b) The complete decompositions of the signal by EMD; imf1 is the first component, and res is the residual.

intermittent and its scale is comparatively small, whereas s 2 is a simple sinusoidal signal with a larger scale. According to the EMD theory, several sifting processes are necessary to extract an IMF. Figure A2 $(a)$ is the result after sifting once. We can see that there are many oscillations with a larger scale left in the result shown in the third row of figure A2(a); however only one sifting process was carried out. Figure A2(b) shows the final decomposition of the signal into imf1 and a residual (res). Again there are still some oscillations of small scale mixed with large scale in imf1, and this is the first type of scale mixing described above; oscillations of large scale also exist in both imf 1 and the residual — this is the second type of scale mixing; finally, at the location denoted as (1), because of the relatively small value of imf 1 , the corresponding location of the residual is relatively large, and at location (2) the opposite situation occurs - this is the third type of scale mixing.

Figure A3 shows the decomposition of the same signal shown in figure A2, using EEMD. Figure A3(a) shows the result when the ensemble number is set to 1 . Without stacking, the first three IMFs contain much noise besides the signal, but the purpose of avoiding scale mixing has been achieved for there are no oscillations of large scale in the first three IMFs. However, the result does not look so good without stacking. Figure $\mathrm{A} 3(b)$ is the result when the ensemble number is set to 100 . Now the result looks much better because the noise is nearly completely eliminated by stacking. In order to obtain a result with more physical meaning, the first three IMFs were combined into a new component $\mathrm{c} 1$, and the last two IMFs into another component $\mathrm{c} 2$, as shown in figure $\mathrm{A} 3(c)$. Figure A3 $(d)$ shows the differences between the original signal and the results from EEMD (diff1 is the result of s1 minus $\mathrm{c} 1$ and diff2 is the result of s2 minus c2). The locations marked by circles are those which earlier had relatively large differences, but that now look very small, confirming the excellent result obtained with EEMD.

\section{Appendix B. Sensitivity of EMD to perturbations}

Any digital signal processing method should be stable and robust, and therefore one does not expect very large changes in the result from just tiny oscillations in the input signal. However, the performance of EMD is not satisfactory in this respect (Wu and Huang 2009, Wu et al 2009). In the smooth area of a signal, if a tiny change is introduced at any point, the decompositions will show dramatic changes in a broad range centred at the perturbed point.

In the smooth area of the signal, there are a few extrema and therefore the interpolated envelopes can easily be significantly influenced by the 'outsider' perturbation. Because of such tiny perturbations, there will appear one or more extrema. Since the envelopes are interpolated from the extrema, the envelopes will change a lot around the perturbations, which will in turn cause the mean of envelopes to also change a lot, and this will affect the final decompositions. The affected area will be mainly between the two extrema closest to the perturbations, because when applying cubic splines, the area between two nodes (here referred to as extrema) is most affected by the two closest nodes; the other nodes will have less and less influence with increasing distance from the perturbation. The reason why EEMD can reduce sensitivity to perturbations is that the added white noise make the extrema distributed along the signal, and therefore the area affected by the local perturbation will be minimized.

Another example is shown in figure B1. Here, the original signal is now composed of the sum of two sinusoidal signals. Figure B1 (a) shows the resulting EMD decomposition. Because of the lack of adequate sampling rate and the influence of edge effects, the decompositions are not identical to the original sinusoidal signal, especially near the boundaries. Nevertheless, the overall pattern is fairly similar, and it was possible to successfully decompose the signal into two components. In figure B1(b), a small perturbation was added at point 100 in the horizontal axis (marked by the circle), and then the signal was again decomposed with EMD. From the 
(a)

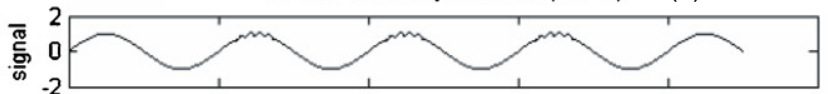

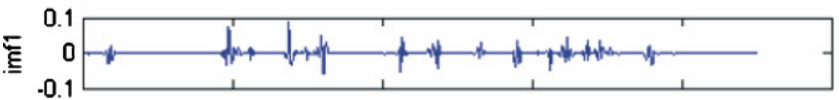

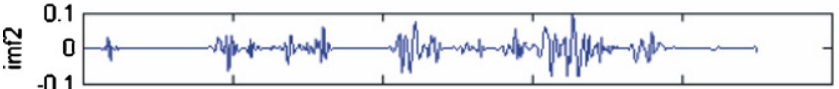

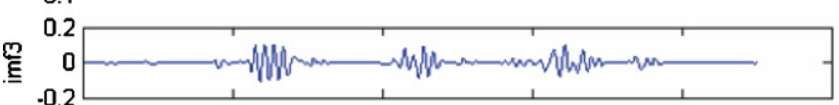
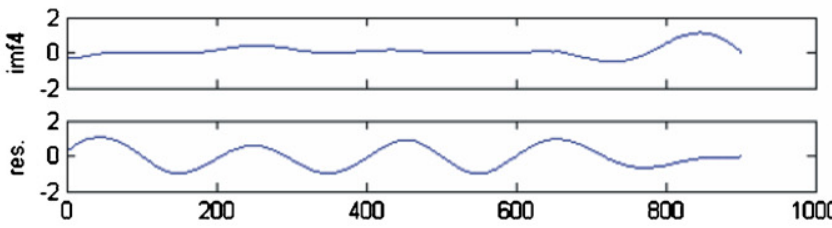

Combined EEMD Decompositions (c)
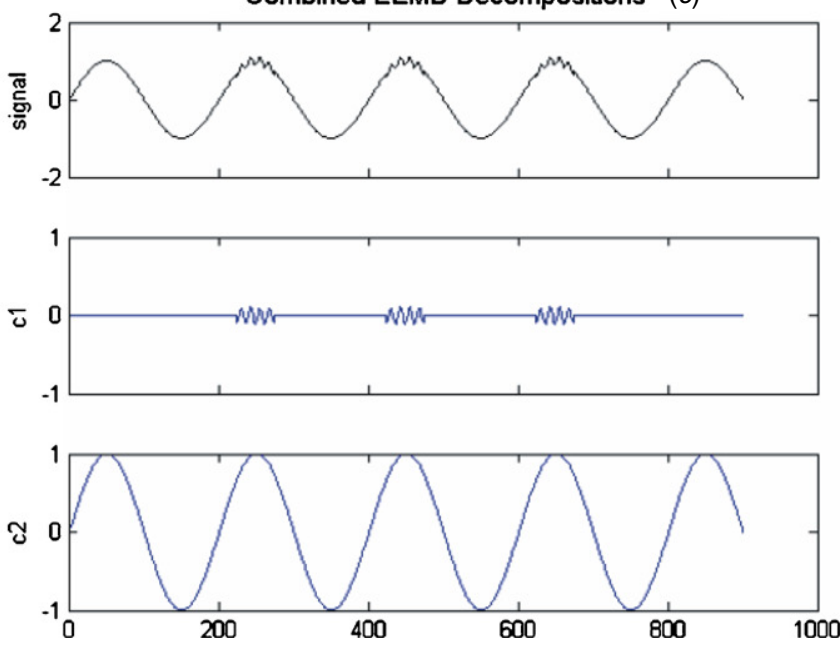

$(b)$
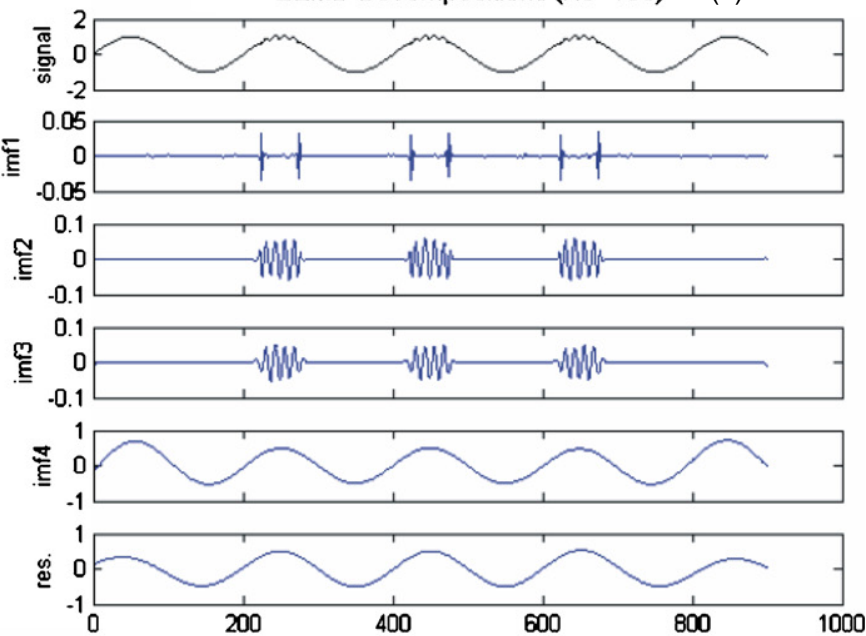

Difference

(d)
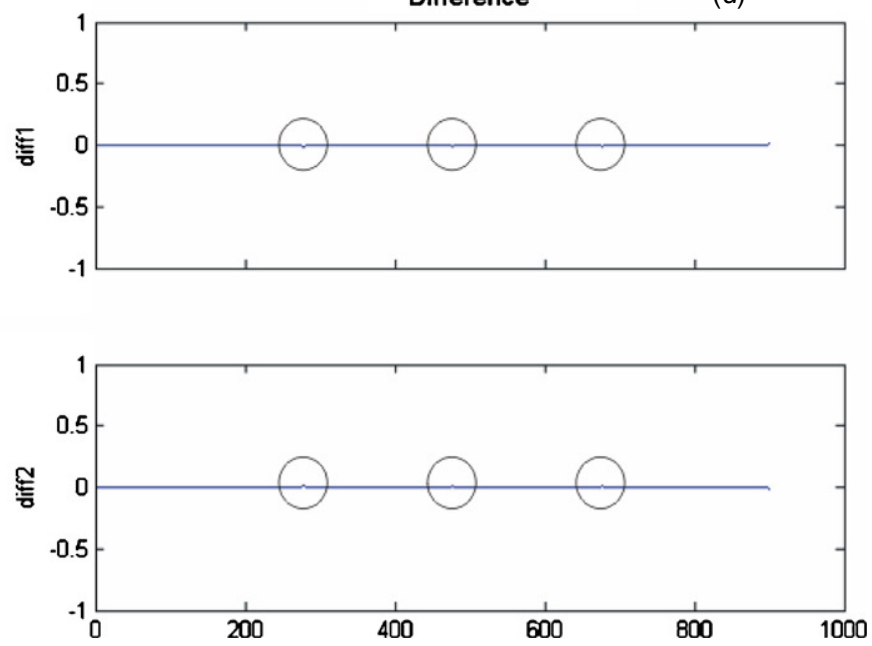

Figure A3. (a) EEMD decomposition of the synthetic signal shown in figure A1 for an ensemble number of 1 (NE $=1$ ). ( $b$ ) EEMD decomposition for $\mathrm{NE}=100 .(c) \mathrm{c} 1$ is the sum of $\mathrm{imf} 1$, imf 2 and imf 3 ; $\mathrm{c} 2$ is the sum of imf 4 and the residual. $(d)$ diff 1 is the difference between $\mathrm{c} 1$ and $\mathrm{s} 1$ (of figure A1); diff 2 is the difference between $\mathrm{c} 2$ and $\mathrm{s} 2$. The three circles mark the positions where some very small differences can be observed.
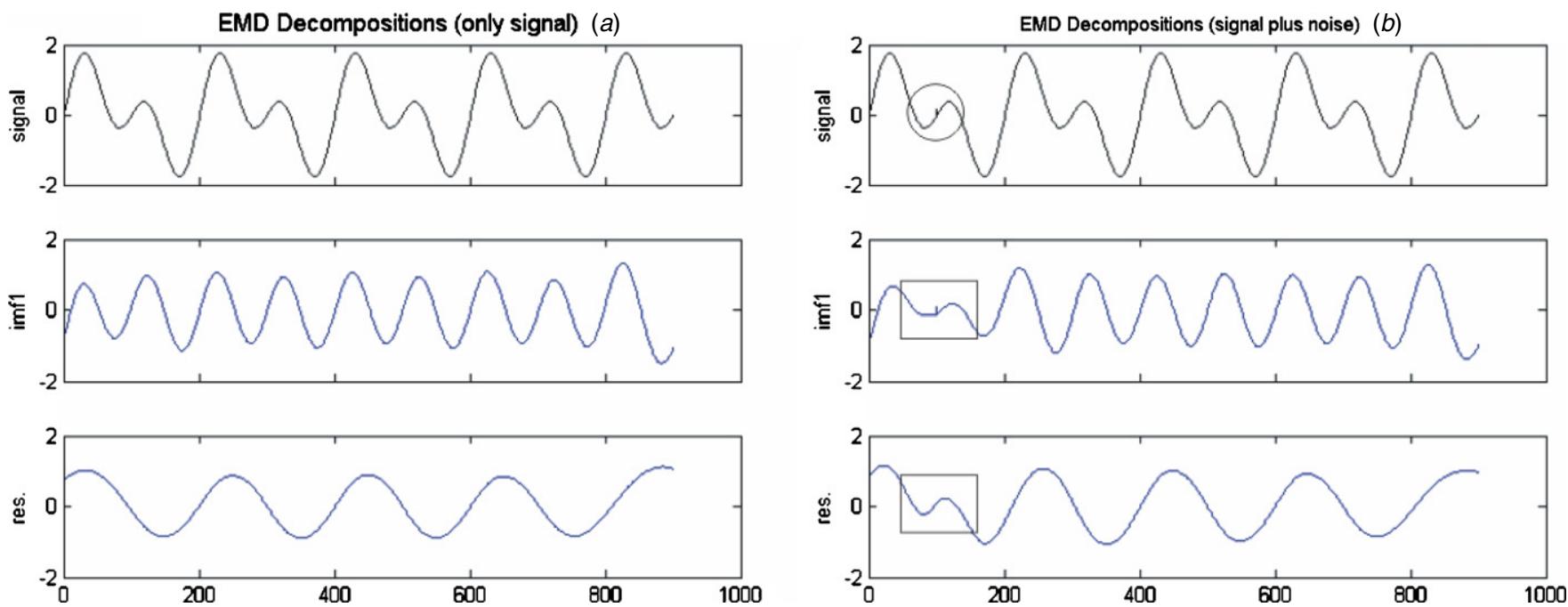

Figure B1. Synthetic signal composed of the sum of two sinusoidal curves. (a) The decompositions by EMD; $(b)$ the EMD decomposition when there is a small disturbance added at the position marked by the circle. Rectangles roughly mark the areas affected by the disturbance. 

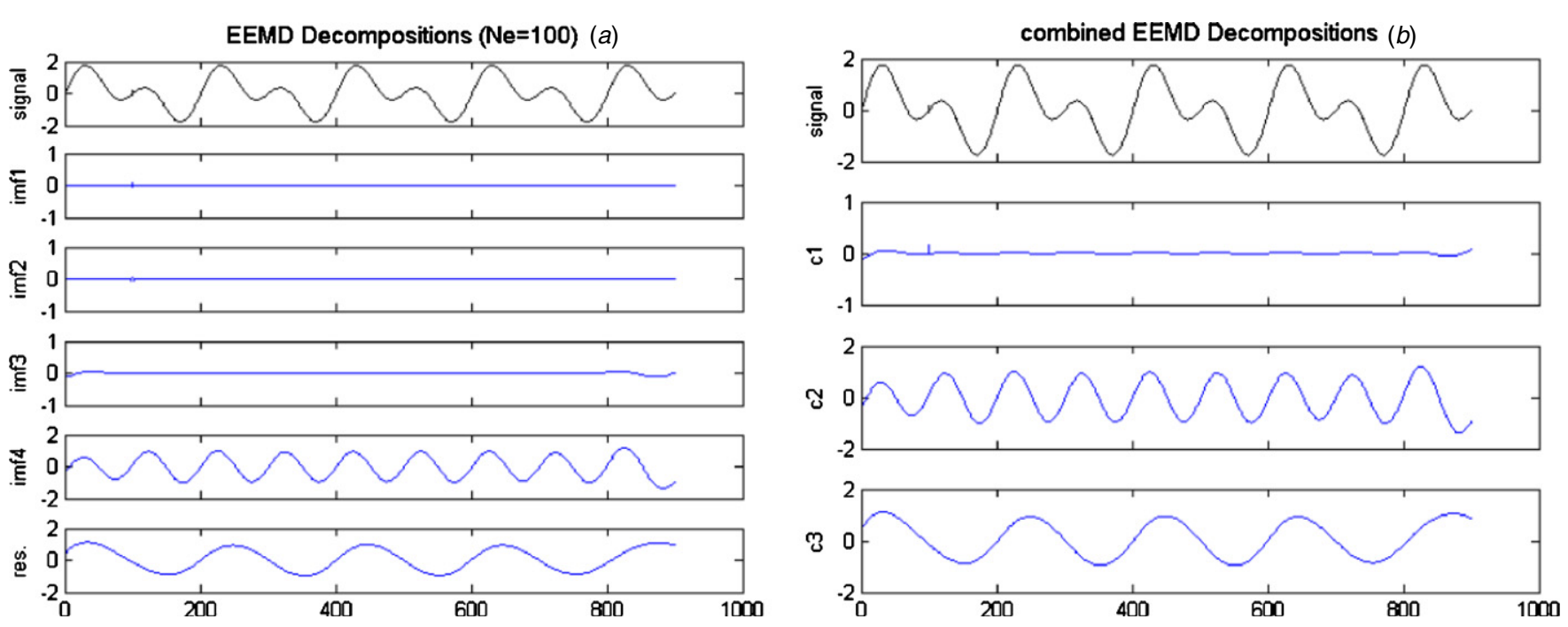

Figure B2. (a) EEMD decompositions of the signal in figure $\mathrm{B} 1(b)$, for $\mathrm{NE}=100 ;(b) \mathrm{c} 1$ is the sum of imf1, imf 2 and imf $3 ; \mathrm{c} 2$ is imf4; 3 is the residual.

next two images of figure B1 $(b)$ it can be observed that the results of decompositions change a lot within the area marked by the rectangle. This is what we would like to avoid, and is due to the sensitivity of EMD to perturbations, rather than related to any real physical meaning of the signal. Figure B2 shows the decompositions of the signal (same as shown in figure $\mathrm{B} 1(b))$ by EEMD with the ensemble number equal to 100 . Contrary to the results from EMD, not only has the stability of the decomposition process been guaranteed, but also the perturbation could be isolated. As shown in figure $\mathrm{B} 2(a)$, the perturbation was mainly contained in imf1 and imf2; imf3 contains little information of the original signal but has some edge effects. The main components of the signal are contained in imf 4 and the residual, respectively. Just as before, the first three IMFs were combined into a new component $\mathrm{c} 1$, imf 4 was treated as a new component $\mathrm{c} 2$, and the residual was treated as c3 (figure B2(b)).

\section{References}

Cai S Q and Gan Z J 2001 Progress in the study of the internal solition in the northern South China Sea Adv. Earth Sci. 16 215-9 (in Chinese)

Dong C Z, Song H B, Hao T Y, Chen L and Song Y 2009 Studying of oceanic internal wave spectra in the northeast South China Seas from seismic reflections Chin. J. Geophys. 52 2050-5 (in Chinese)

Fang X H and Du T 2005 Fundamental of Oceanic Waves and Internal Waves in the China Seas (Qingdao: China Ocean University Press) in Chinese

Flandrin P, Rilling G and Goncalves P 2004 Empirical mode decomposition as a filter bank IEEE Signal Process. Lett. $11112-4$

Garrett C and Munk W 1972 Space-time scales of internal waves Geophys. Astrophys. Fluid Dyn. 3 225-64

Garrett C and Munk W 1975 Space-time scales of internal waves: a progress report J. Geophys. Res. 80 291-7
Garrett C and Munk W 1979 Internal waves in the ocean Annu. Rev. Fluid Mech. 11 339-69

Holbrook W S and Fer I 2005 Ocean internal wave spectra inferred from seismic reflection transects Geophys. Res. Lett. 32 L15604

Holbrook W S, Paramo P, Pearse S and Schmitt R W 2003 Thermohaline fine structure in an oceanographic front from seismic reflection profiling Science 301 821-4

Huang N E, Shen Z and Long S R 1999 A new view of nonlinear water waves- the Hilbert spectrum Annu. Rev. Fluid Mech. 31 417-57

Huang N E, Shen Z, Long S R, Wu M C, Shin H H, Zheng Q A, Yen N C, Tung C C and Liu H H 1998 The empirical mode decomposition and the Hilbert spectrum for nonlinear and non-stationary time series analysis Proc. R. Soc. A 454 903-95

Jiang G R, Zhang J, Shi W L and Liu Q Y 2009 The Ocean Internal Waves and its Influence on Naval Battle (Beijing: China Meteorological Press) in Chinese

Liu A K, Chang Y S and Nai K L 1998 Evolution of nonlinear internal waves in the East and South China seas J. Geophys. Res. 103 7995-8008

Song H B, Bai Y, Dong C Z and Song Y 2010 A preliminary study of application of empirical mode decomposition method in understanding the features of internal waves in the northeastern South China Sea Chin. J. Geophys. 53 393-400 (in Chinese)

Song H B, Dong C Z, Chen L and Song Y 2008 Reflection seismic methods for studying physical oceanography: introduction of seismic oceanography Prog. Geophys. 23 1156-64 (in Chinese)

Song H B, Pinheiro L, Wang D X, Dong C Z, Song Y and Bai Y 2009 Seismic images of ocean meso scale eddies and internal waves Chin. J. Geophys. 52 2775-80 (in Chinese)

Wu Z H and Huang N E 2004 A study of the characteristics of white noise using the empirical mode decomposition method Proc. $R$. Soc. A 460 1597-611

Wu Z H and Huang N E 2009 Ensemble empirical mode decomposition: a noise-assisted data analysis method $A d v$. Adapt. Data Anal. 1 1-41

Wu Z H, Huang N E and Chen X Y 2009 The multi-dimensional ensemble empirical mode decomposition method Adv. Adapt. Data Anal. 1339-72 
Analysis of ocean internal waves imaged by multichannel reflection seismics, using ensemble empirical mode decomposition

This content has been downloaded from IOPscience. Please scroll down to see the full text.

2012 J. Geophys. Eng. 9302

(http://iopscience.iop.org/1742-2140/9/3/302)

View the table of contents for this issue, or go to the journal homepage for more

Download details:

IP Address: 193.137.168.194

This content was downloaded on 27/09/2016 at 16:24

Please note that terms and conditions apply.

You may also be interested in:

Determination of knock characteristics in spark ignition engines: an approach based on ensemble empirical mode decomposition

Ning Li, Jianguo Yang, Rui Zhou et al.

Removal of artifacts in knee joint vibroarthrographic signals using ensemble empirical mode

decomposition and detrended fluctuation analysis

Yunfeng Wu, Shanshan Yang, Fang Zheng et al.

Detecting the bonding state of explosive welding structures based on EEMD and sensitive IMF time entropy

Yue Si, Zhousuo Zhang, Qiang Liu et al.

Fault diagnosis of rotating machinery using an improved HHT based on EEMD and sensitive IMFs

Yaguo Lei and Ming J Zuo

Analysis of Solar Coronal Oscillations

J. Terradas, R. Oliver and J. L. Ballester

Rotation Residuals of Solar Convection Zone

R. W. Komm, F. Hill and R. Howe 


\title{
Analysis of ocean internal waves imaged by multichannel reflection seismics, using ensemble empirical mode decomposition
}

\author{
Haibin Song ${ }^{1}$, Yang Bai ${ }^{1,2}$, Luis Pinheiro ${ }^{3}$, Chongzhi Dong ${ }^{4}$, \\ Xinghui Huang ${ }^{1,2}$ and Boran Liu ${ }^{1,2}$ \\ ${ }^{1}$ Key Laboratory of Petroleum Resources Research, Institute of Geology and Geophysics, \\ Chinese Academy of Sciences, Beijing 100029, People's Republic of China \\ ${ }^{2}$ Graduate University of Chinese Academy of Sciences, Beijing 100049, People's Republic of China \\ ${ }^{3}$ Departamento de Geociências and CESAM, Universidade de Aveiro, 3800 Aveiro, Portugal \\ ${ }^{4}$ Key Laboratory of Submarine Geosciences, Second Institute of Oceanography, Hangzhou 310012, \\ People's Republic of China \\ E-mail: baiyang@mail.iggcas.ac.cn
}

Received 1 September 2011

Accepted for publication 3 April 2012

Published 26 April 2012

Online at stacks.iop.org/JGE/9/302

\begin{abstract}
Research on ocean internal waves using seismic oceanography is a frontier issue both for marine geophysicists and physical oceanographers. Images of the ocean water layer obtained by conventional processing of multichannel seismic reflection data can show the overall patterns of internal waves. However, in order to extract more information from the seismic data, new tools need to be developed. Here, we use the ensemble empirical mode decomposition (EEMD) method to decompose vertical displacement data from seismic sections and apply this method to a seismic section from the northeastern South China Sea, where clear internal waves are observed. Compared with the conventional empirical mode decomposition method, EEMD has greatly reduced the scale mixing problems induced in the decomposition results. The results obtained show that the internal waves in this area are composed of different characteristic wavelengths at different depths. The depth range of 200-1050 m contains internal waves with a wavelength of $1.25 \mathrm{~km}$ that are very well coupled in the vertical direction. The internal waves with a wavelength of $3 \mathrm{~km}$, in the depth range of 200-600 m, are also well coupled, but in an oblique direction; this suggests that the propagation speed of internal waves of this scale changes with depth in this area. Finally, the internal waves with a wavelength of $6.5 \mathrm{~km}$, observed in the depth range of 200-800 m, are separated into two parts with a phase difference of about $90^{\circ}$, by a clear interface at a depth of $650 \mathrm{~m}$; this allows us to infer an oblique propagation of wave energy of this scale.
\end{abstract}

Keywords: seismic oceanography, ocean internal waves, EMD, EEMD

(Some figures may appear in colour only in the online journal)

\section{Introduction}

Ocean internal waves are waves that oscillate within, rather than at the surface of seawater when there is density stratification. The frequency of ocean internal waves varies between the inertial frequency and the Brunt-Vaisala frequency. Ocean internal waves are the most important part of the ocean energy cascade. The generation, propagation and dissipation of ocean internal waves cause great energy exchange with an important influence on the ocean dynamic processes. Unlike surface waves, the amplitude of internal waves can be very large, sometimes greater than $100 \mathrm{~m}$ (Liu 
et al 1998); therefore they can have major impacts on the safety of marine engineering, ocean communications, oil exploration (Cai and Gan 2001) or marine military facilities (Jiang et al 2009). Therefore, the research on ocean internal waves is of great concern to a large number of scientists from various countries.

The development of research on ocean internal waves is however highly dependent on the quality and quantity of observational data. Since the early 1970s, with the rapid development of oceanography, the intense development of new instrumentation tools to probe the oceans allowed great progress to be made in research on ocean internal waves. Garrett and Munk (1972) proposed the GM72 model spectra for ocean internal waves, and through constant improvement, new editions of this model have been proposed subsequently (Garrett and Munk 1975, 1979), which are also referred to as GM model spectra. Although these spectra are essentially statistical descriptions of oceanic phenomena, they have been a key reference in analysing the data from ocean internal waves. One of the limitations of conventional physical oceanography instrumentation, such as XBTs (expendable bathythermographs), CTDs (conductivity, temperature and depth sensors), XCTDs (expendable CTDs) and ADCPs (acoustic Doppler current meters), is that they cannot provide vertical sections of the ocean in a very short time and lack good lateral resolution. Remote sensing cannot also provide the necessary subsurface water information directly (Fang and Du 2005).

In 2003, Holbrook showed that there were weak reflection signals inside the water that could be correlated with the ocean structure (Holbrook et al 2003); this gave rise to an exciting new research field: seismic oceanography. Holbrook and Fer (2005) further noted that there were continuous undulations in the reflection section of seawater. They compared the spectra of these undulations with the GM76 model spectra, and proposed that these undulations were representations of ocean internal waves, hence laying the foundations for the research on ocean internal waves using the multichannel seismic reflection method. Compared with conventional oceanography measurements, seismic reflection can provide vertical sections of the water layer in a very short time, with the required high lateral resolution to image internal waves.

The northeastern South China Sea is an excellent internationally recognized natural laboratory for these types of studies (Fang and Du 2005). Song et al (2009) processed a seismic section from the northeast South China Sea and showed that the undulations of the reflections in the stacked seismic section exhibited the overall patterns of internal waves. Dong et al (2009) computed the wavenumber spectra ${ }^{5}$ of these undulations and found that the overall patterns of those spectra were very similar to the GM76 model spectra. More recently, Song et al (2010) decomposed part of that section using the EMD (empirical mode decomposition) method Huang et al (1998), with encouraging results. However, recent studies showed that, although useful, the EMD method has some

\footnotetext{
5 Since the signals analysed here are a function of distance (space) and not time, therefore instead of frequency spectrum we will always use the term wavenumber spectrum.
}

severe drawbacks, such as scale mixing problems (Huang et al 1999), which cause some difficulties in the physical analysis of the results. In order to overcome the drawbacks of EMD, Wu and Huang (2009) more recently proposed an improved method called ensemble EMD (EEMD). This paper investigates the characteristics of ocean internal waves in northeastern South China Sea using EEMD.

\section{The EMD method and its drawbacks}

Unlike almost all previous signal analysis methods, the EMD method is adaptive. Huang et al (1999) have shown that all signals can be interpreted as composed of a series of IMFs (intrinsic mode functions) and the objective of EMD is precisely to decompose signals into series of IMFs and a residual (1):

$$
\mathbf{X}(t)=\sum_{i=1}^{n} \mathbf{c}_{i}+\mathbf{r}_{n},
$$

where $\mathbf{X}(t)$ is the signal to be decomposed, $\mathbf{c}_{i}$ are the resultant IMFs and $\mathbf{r}_{n}$ are residuals.

IMFs are oscillatory functions with varying amplitude and frequency, which are defined as follows: (1) throughout the whole length of an IMF set, the number of extrema (including all maximum and minimum) and the number of zero-crossings must either be equal or differ at most by $1 ;(2)$ at any data location, the mean value of the envelope defined by the local maxima and the envelope defined by the local minima is zero (actually, it is not strictly zero but controlled by a certain stop criterion).

In practice, the IMFs are obtained through a sifting process.

(1) Firstly, all the local maxima are identified and connected with cubic splines to obtain the upper envelope of the signal (the length of the envelope should be the same as the original data set); this process is then repeated for the local minima to obtain the lower envelope of the signal (the whole data set variation should then be within the range between the two envelopes).

(2) Secondly, the mean of the upper and lower envelopes $\left(\mathbf{m}_{1}\right)$ is calculated and the difference $\left(\mathbf{h}_{1}\right)$ between the original data and $\mathbf{m}_{1}$ determined:

$$
\mathbf{X}(t)-\mathbf{m}_{1}=\mathbf{h}_{1} .
$$

This process is known as the 'one sifting process'; ideally, $\mathbf{h}_{1}$ should be an IMF, but this is not usually the case. Consequently, the sifting process should be repeated several times until the results satisfy the definitions of IMFs

$$
\begin{gathered}
\mathbf{h}_{1}-\mathbf{m}_{11}=\mathbf{h}_{11}, \quad \mathbf{h}_{11}-\mathbf{m}_{12}=\mathbf{h}_{12} \ldots \\
\mathbf{h}_{1(k-1)}-\mathbf{m}_{1 k}=\mathbf{h}_{1 k} .
\end{gathered}
$$

(3) Then, denoting the final result $\left(\mathbf{h}_{1 k}\right)$ by $\mathbf{c}_{1}$ and the residual $\left(\mathbf{X}(t)-\mathbf{c}_{1}\right)$ by $\mathbf{r}_{1}, \mathbf{c}_{1}$ is the first component while $\mathbf{r}_{1}$ is now treated as the data and the decomposition process is repeated again and again so as to obtain $\mathbf{c}_{2}, \mathbf{c}_{3}, \ldots, \mathbf{c}_{n}$ in turn, and a residual $\mathbf{r}_{n}$.

In terms of its practical application, the EMD method mainly has the following two main drawbacks: (1) sometimes, there will be scale mixing problems in some IMFs; (2) the EMD is too sensitive to some local tiny changes. 


\section{The EEMD method}

In order to overcome some of the drawbacks of EMD, Wu and Huang (2009) proposed an improved method, called EEMD. Based on EMD, the EEMD method improves the results of the decomposition through the following two techniques: (1) noise-assisted data analysis, i.e. the addition of finite amplitude (usually small) white noise to the data before decomposition; (2) the application of a simple statistics principle-stacking the white noise many times will suppress that noise.

If the wavenumber spectra are continuous (i.e. if they have a continuous representation of all constituting components of different scales), the centre wavenumber of the spectra of the successive IMFs, obtained through the EMD sifting process, will be approximately halved (Wu and Huang 2004, Flandrin et al 2004). However, when the wavenumber spectrum is not continuous, although the centre wavenumber for each successive IMF spectra will still decrease, there are no fixed rules in this case, and this often causes scale mixing problems in the data analysis, especially when processing $2 \mathrm{D}$ data sets (such as images or seismic sections). For 2D data sets, such as the one analysed here, that result from the picking of the main reflectors in the horizontal direction from a seismic section (see figure 1), these are treated as composed of a vertical stack of 1D data sets, each of which is decomposed in the horizontal direction.

What often happens is that the information from similar scales is distributed in different levels of IMFs (i.e. the information concerning a certain scale can be distributed between the second and third level IMFs) and/or IMFs of a certain level contain information from various different scales. However, when we work with images and combine the IMFs, we usually combine the IMFs of the same level as one component (of the image). For example, we combine all the IMFs of the second level as one component, and all the IMFs of the third level as another component. If in one of the components there is information from different scales, then there is scale mixing. Sometimes, scale mixing problems can be avoided by adjusting some IMFs manually; however, when the data volume is too large, this will be inefficient and, moreover, when a single IMF has scale mixing problems it is meaningless to do any adjustment.

However, when we apply the EEMD method ( $\mathrm{Wu}$ and Huang 2009), we will first add finite amplitude white noise to the data (with the same length as the data set). This will make the wavenumber spectrum energy (maybe not continuous originally) more continuous, because the added energy of the white noise will be evenly distributed throughout the wavenumber spectrum. In such a case, the ranges of wavenumber spectra of the successive IMFs will again decrease by a factor of about 2 (Wu and Huang 2009). This will then reduce the scale mixing problems.

Although we can reduce the scale mixing problems by adding white noise to the data, the result of the decompositions may not look good because of the added noise; however, we can use the stacking technique, mentioned above, to reduce or cancel significantly the added noise. We repeat the noiseadded decompositions a certain number of times (called the ensemble number-NE), with different white noise added each time; then we stack and average the IMFs of the same level, to suppress the added white noise (since the addition of many white noise sequences will statistically tend to cancel each other). Theoretically, if the ensemble number approaches infinity, the white noise will be fully suppressed to zero. However, in practice, stacking for about 100 times is generally good enough. It should be noted that, because of the added noise, sometimes there are IMFs containing no information on the original data since they purely consist of noise; these IMFs will generally approach zero after stacking.

In summary, the implementation steps for EEMD are as follows (Wu and Huang 2009).

(1) Add white noise to the data and apply EMD.

(2) Subtract the same white noise used by step (1) from the data, and apply EMD.

(3) Stack the results of steps (1) and (2).

(4) Repeat steps (1)-(3) several times (NE times) with different white noise added each time.

(5) Stack all the IMFs of the same level and divide the result by $2 \times \mathrm{NE}$, to obtain the average.

The purpose of steps (1) and (2) is to suppress the white noise more efficiently with a certain finite number of stacks. Some points should nevertheless be noted: (1) sometimes IMFs of different levels should be combined to obtain a result with more physical meaning (see the discussion above); (2) frequently, it is also not necessary to decompose the data until it cannot be decomposed any further; we can decompose as we need, and treat the remaining as a residual; however, it should be noted that the number of IMFs should not exceed $\left\lfloor\log _{2}(N)\right.$ $-1\rfloor$, where $N$ is the length of data, i.e. the number of samples, and \lfloor\rfloor denotes the floor function ${ }^{6}$ of the expression inside; (3) theoretically, we can suppress any finite amplitude white noise by performing an infinite number of stacks; however, since the ensemble number is finite and we aim to highlight small undulations in the data, it is better to add white noise with only a small energy.

Appendix A and appendix B give some detailed discussions and illustrations of how EEMD can solve the two main drawbacks that EMD has.

\section{Application of EEMD to the analysis of ocean internal waves}

Song et al (2010) processed a seismic section (figure 1) from the northeastern South China Sea, and got the vertical displacement distribution of internal waves shown in figure 2. These authors then decomposed figure 2 in the common mid point (CMP) direction (horizontal), using the EMD method; from the resulting low wavenumber components they were able to image the fine structure of the internal waves, as shown in figure 3, and get new insights into their structure and kinematics. Figure 3(a) shows 10-11 positive and negative vertical displacement bands, well coupled in the vertical direction in

6 The floor function of $x$, also called the greatest integer function or integer value, gives the largest integer less than or equal to $x$. 


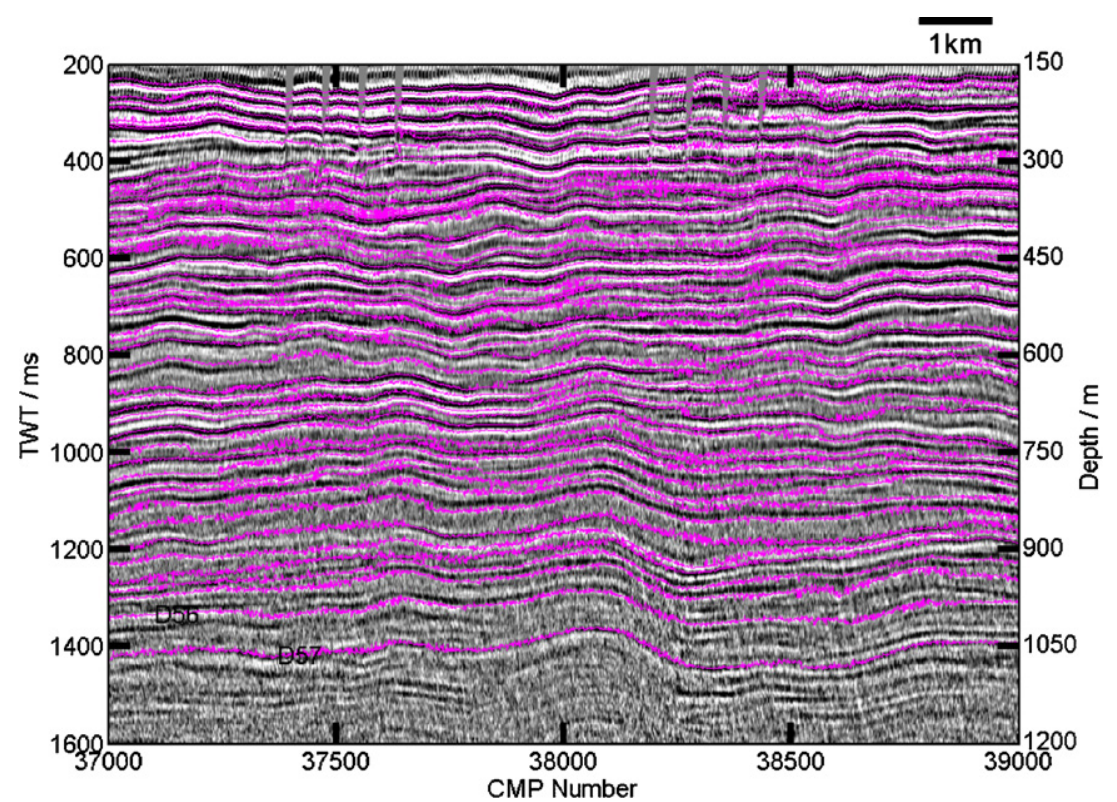

Figure 1. Seismic reflection section of the water column from the northeastern South China Sea (Song et al 2010). The purple lines denote the picked horizons (such as D56 and D57). TWT is the two-way travel time; CMP stands for common mid point.

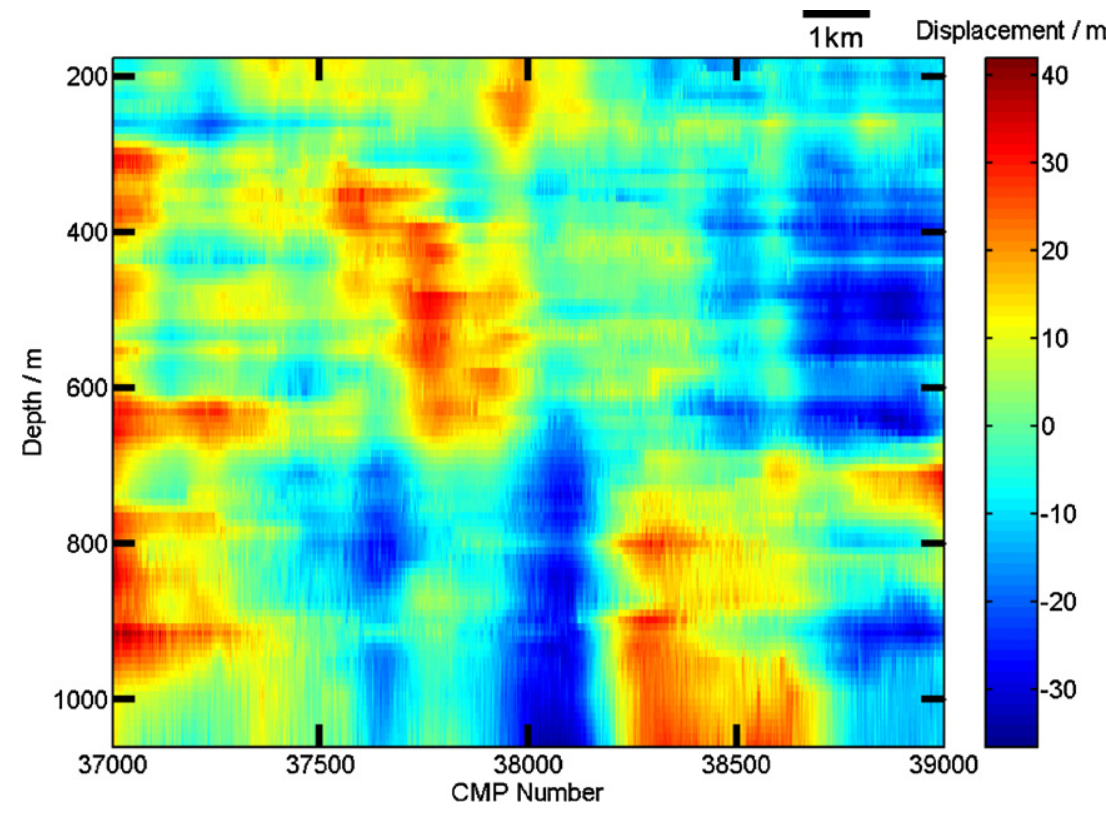

Figure 2. The vertical displacement distribution of internal waves computed from figure 1, by subtracting the trend from each picked horizon in the original data.

the depth range of 200-900 $\mathrm{m}$. Figure 3(b) shows 5-6 positive and negative vertical displacement bands, well coupled in the vertical direction in the depth range of $600-1000 \mathrm{~m}$. The waves shown in figures $3(c)$ and $(d)$, however, show a large difference between the upper and lower areas, separated by clear interfaces at 600 and $700 \mathrm{~m}$, respectively. In particular for figure $3(d)$, the phase difference is about $90^{\circ}$, which was interpreted as evidence of oblique propagation of the wave energy.

A more careful analysis, however, shows that the four components obtained with EMD have evidence of scale mixing. In figure $3(a)$ the scales of areas (1) and (2) are similar to the overall scale of figure $3(b)$. In figure $3(b)$, the scales of area (4), denoted by four rectangles, are also similar to the overall scale of figure $3(a)$, and the pattern of area (3) is almost the same as that of area (6) in figure $3(c)$. As such, it appears better to incorporate area (5) in figure $3(b)$ into the corresponding place in figure $3(c)$, and to incorporate area (7) in figure 3(c) into the corresponding place in figure 3(d). This brief analysis shows that figure 3(b) clearly has various scale mixing problems, almost everywhere. Also, there are many 'stratified' patterns in the four components, such as the lower part of area (3), in figure 3(b). This is also a type of scale mixing, and the missing part of the 'stratified' patterns can always be found in the neighbouring components. The existence of these scale mixing problems has greatly affected 

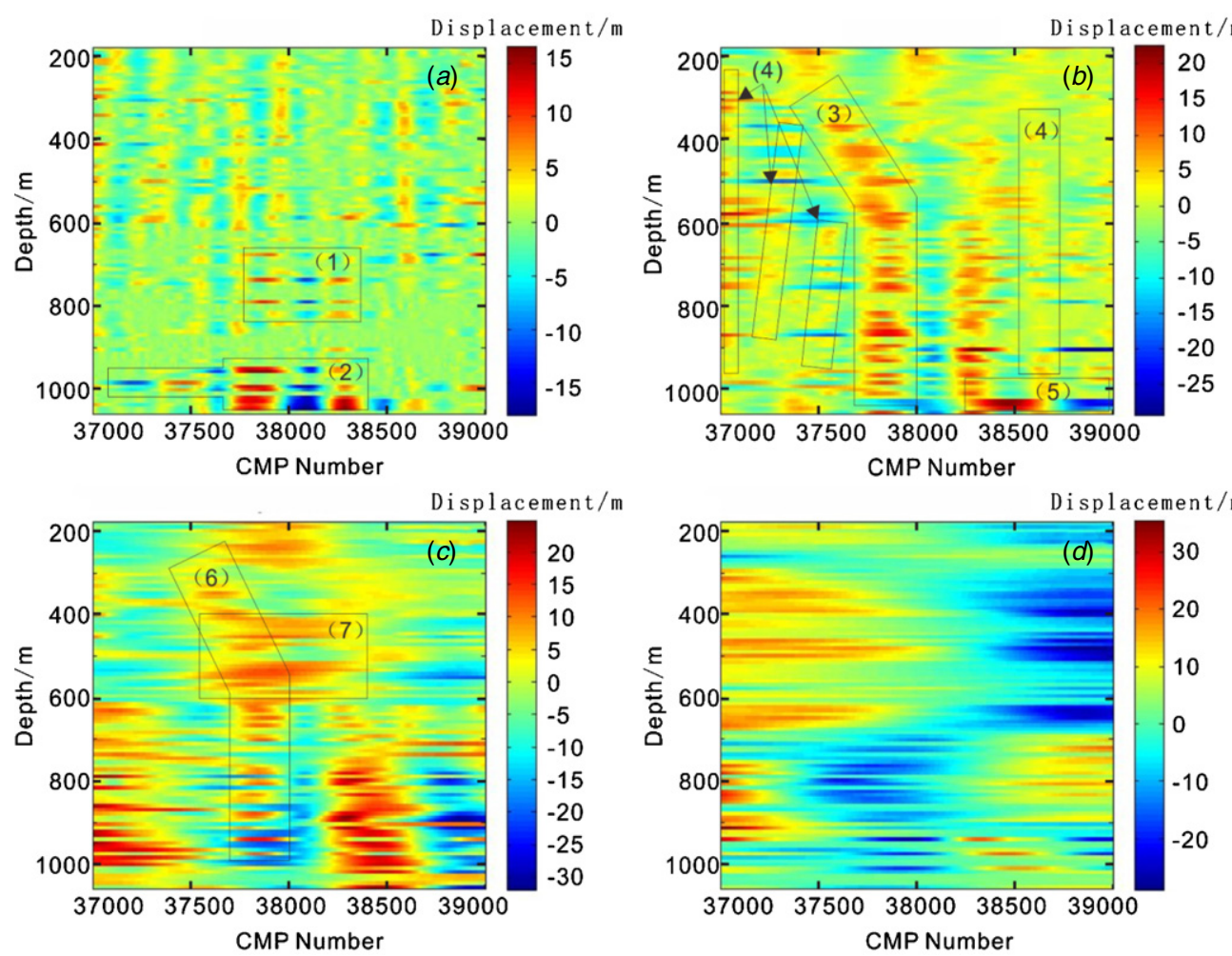

Figure 3. The four low wavenumber components of the EMD decomposition of figure 2: (a) shows 10-11 positive and negative vertical displacement bands well coupled in the vertical direction, while $(b)$ shows 5-6; $(c)$ and $(d)$ show a large difference between the upper and lower areas, separated by clear interfaces at 600 and $700 \mathrm{~m}$, respectively. Areas marked by rectangles are those with various kinds of scale mixing problems: the scales of areas denoted by (1), (2), (5) and (7) are obviously different from their background scales; area (3) and area (6) almost show the same pattern; the scale of area (4) in $(b)$ is more similar to the overall scale of $(a)$. The 'stratified' patterns in all these four components are also the results of scale mixing.
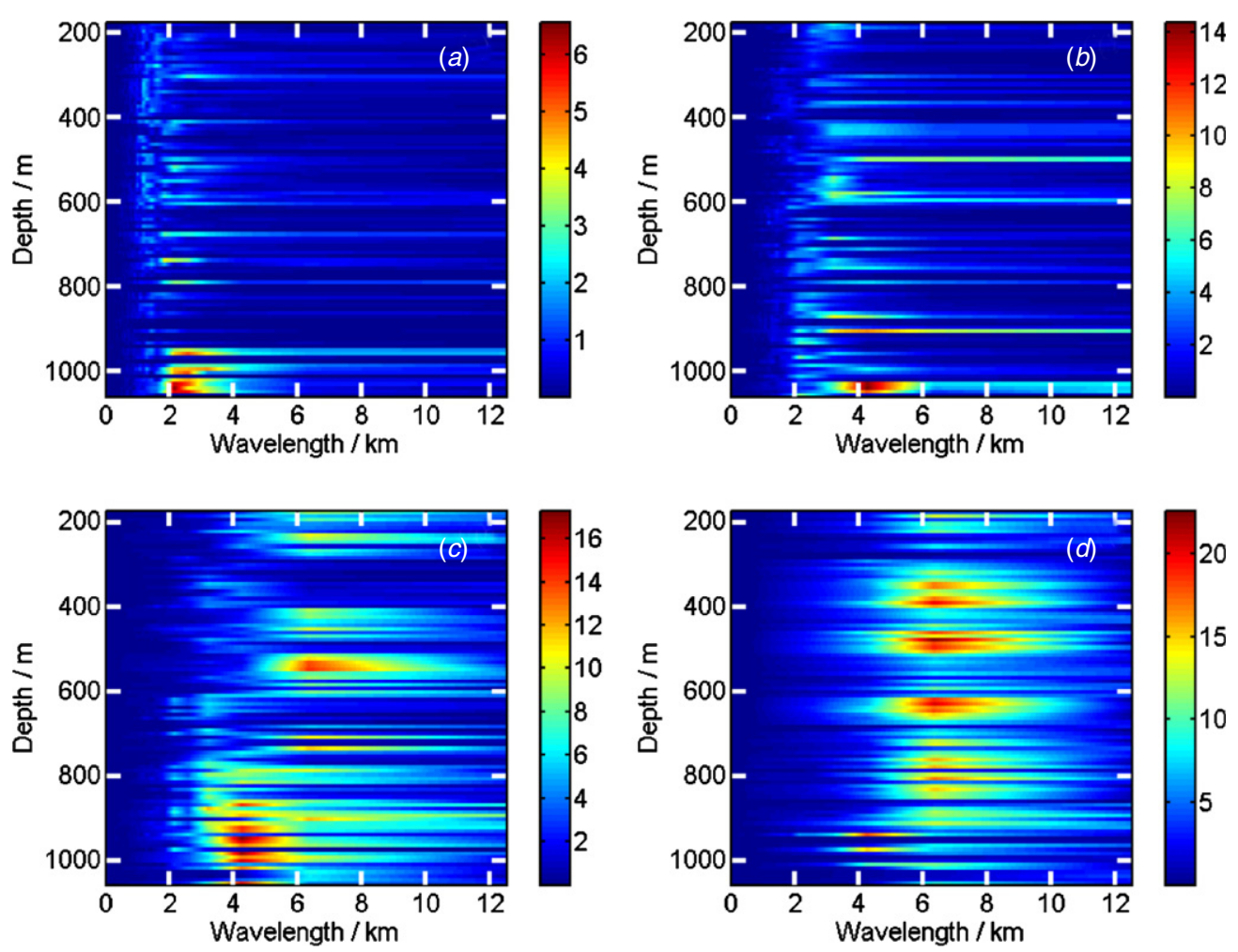

Figure 4. The corresponding wavenumber spectra of figure 3. Affected by the scale mixing problems, all four wavenumber spectra show a 'stratified' pattern, and the wavelength range with significant energy is hard to estimate precisely. 

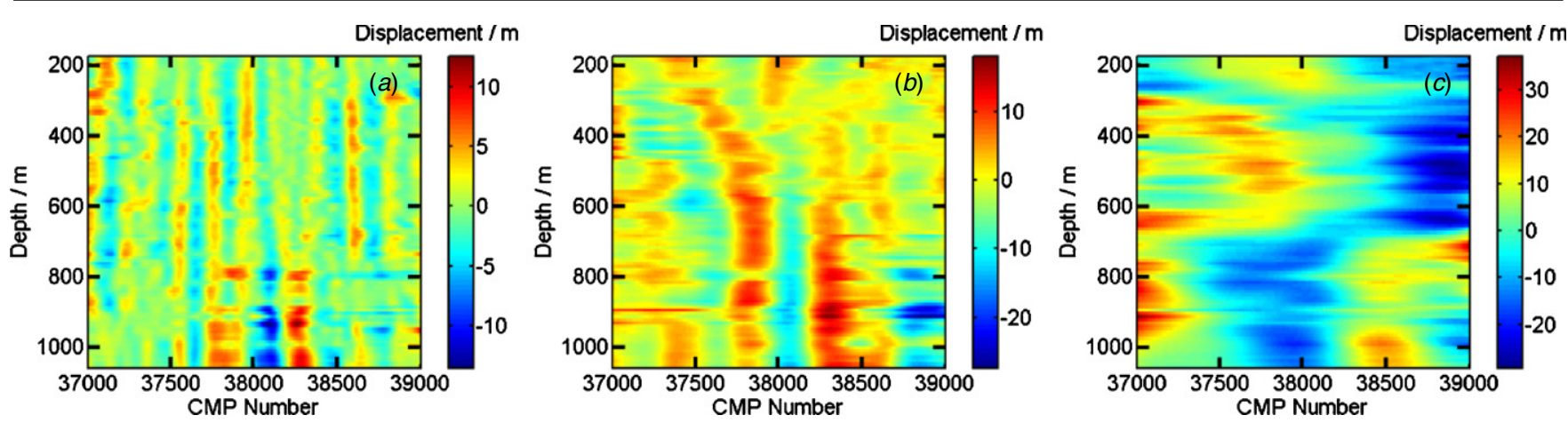

Figure 5. The three low wavenumber components of the EEMD decomposition of figure 2. Compared with figure 3, it is clear that the scale mixing problems are greatly reduced, although some are still present, such as in the middle lower part of $(a)$ and left lower part of $(b)$. The structures shown here are much clearer than those of figure 3.
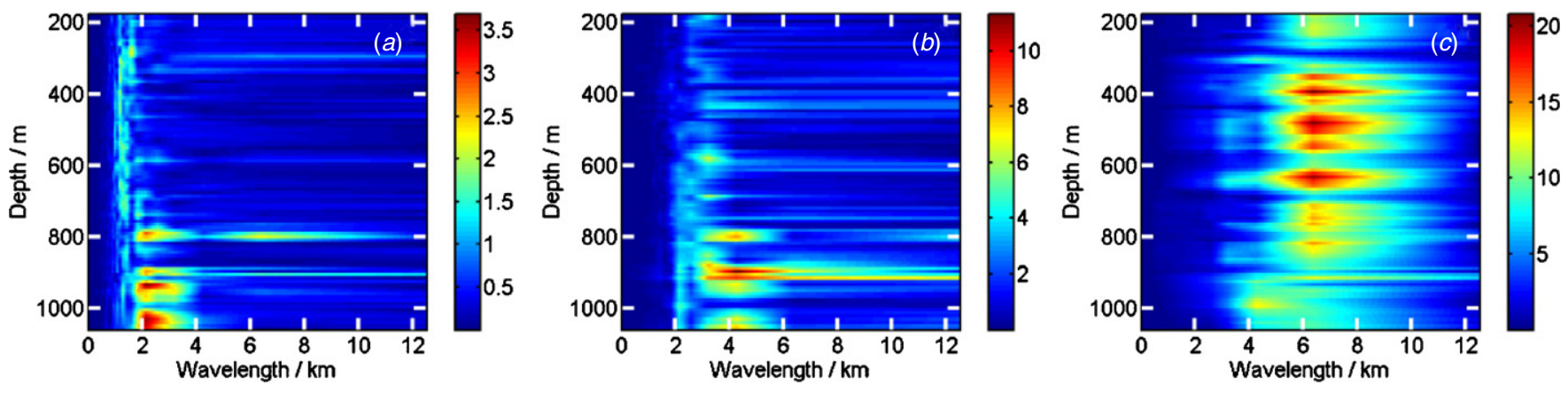

Figure 6. The corresponding wavenumber spectra of figure 5. Due to the reduction of scale mixing problems, the wavelength range for each single component is now much more concentrated than before, and more continuous in the vertical direction. Despite the remaining scale mixing problems in the lower part of $(a)$ and $(b)$, it is still easy to estimate that the characteristic wavelength for $(a)$ is about $1.25 \mathrm{~km}$; for $(b)$, it is about $3 \mathrm{~km}$ for the upper part and $2.2 \mathrm{~km}$ for the lower part; for $(c)$ it is about $6.5 \mathrm{~km}$.

the previous interpretation of the physical meaning of the patterns represented by the four components, especially the estimation of the wavenumber range, and then the estimation of wavelength range (figure 4).

Figure 5 shows the decompositions of figure 2, now using the EEMD method (with the ensemble number NE $=100$ ). Here, it is clear that the main components become three rather than the original four. Compared with figure 3 , it is clear that the scale mixing problems were greatly reduced, although some are still present, such as in the middle lower part of figure 5(a) and left lower part of figure 5(b). With the scale mixing problems greatly reduced, the 'stratified' patterns almost disappeared, revealing a much clearer image of the fine structure of the ocean internal waves. Figure 6 shows the wavenumber spectra corresponding to the three components of figure 5. Comparing figure 6 with figure 4 , due to the reduction of the scale mixing problems, the wavelength range for each single component is now much more concentrated than before, and more continuous in the vertical direction. This provides a much more reliable basis to estimate the wavelength range of each component. From the analysis of the wavenumber spectra, the observed pattern in figure 5(a) is mainly composed of internal waves with a wavelength of $1.25 \mathrm{~km}$, which couple well in the depth range of 200-1050 m. Because there are still some scale mixing problems in the middle lower part, other energy peaks with a wavelength of $2-3 \mathrm{~km}$ in figure $6(a)$, at a depth of about $800 \mathrm{~m}$, and also in the depth range of 900-1000 m, are nevertheless observed. The upper part above
$600 \mathrm{~m}$ in figure $5(b)$ mainly consists of internal waves with a wavelength of $3 \mathrm{~km}$, and the waves in the middle upper part couple well in an oblique direction, with the apparent angle of about $30^{\circ}$ to the vertical direction; from this observation, we infer that the internal waves of this scale, in this part of the section, have different propagating speed with depth. The lower part below $600 \mathrm{~m}$ mainly consists of internal waves with a wavelength of $2.2 \mathrm{~km}$, well coupled in the vertical direction. Like the situation in figure 6(a), there are other energy peaks around $4 \mathrm{~km}$ wavelength in the lower part of the section, at a depth of about $800-900 \mathrm{~m}$, and beneath $1000 \mathrm{~m}$ in figure $6(b)$, in addition to the peaks around the $2.2 \mathrm{~km}$ wavelength. The upper part above $850 \mathrm{~m}$ in figure 5(c) mainly consists of internal waves of $6.5 \mathrm{~km}$ wavelength, while the lower part mainly consists of internal waves of $5 \mathrm{~km}$ wavelength. However, because of the influence of scale mixing, the energy of the lower part in figure $6(c)$ is relatively small. With an interface at $650 \mathrm{~m}$, the upper and lower parts of figure $5(\mathrm{c})$ have a phase difference of about $90^{\circ}$, which can be interpreted as indicative of the oblique propagation of wave energy at this scale.

\section{Conclusions}

The seismic section of the water column obtained from the processing of one multichannel seismic reflection line from the northeastern South China Sea shows undulations in the 
reflectors that likely correspond to the stacking of internal waves of various scales, and therefore can only show their overall pattern. The previous application of the EMD method to this seismic section allowed the extraction of the fine structure of the internal waves, at various scales. However useful the EMD proved to be for this purpose in a first approach, some drawbacks of this method nevertheless remained unsolved, such as scale mixing and sensitivity, which affected the decomposition of the data. As such, in this paper, we applied an advanced version of EMD, called EEMD, to reprocess the same section from the northeastern South China Sea. Comparing the new decompositions with the results previously obtained with EMD we could largely solve the previous scale mixing problems, which are now greatly reduced. The upper part of the section, above a water depth of about $800 \mathrm{~m}$, rarely contains any scale mixing problems. The lower part, however, around the depth of about $800 \mathrm{~m}$ and in the range of 900-1050 $\mathrm{m}$ still has a few remaining scale mixing problems. The resulting wavenumber spectra of the various components now show the energy much more concentrated in the wavenumber range than before, and more continuous in the vertical direction, which provides a much more sound basis to better estimate the wavelength range of each of the components.

The new results show that there are internal waves of $1.25 \mathrm{~km}$ wavelength in the depth range of 200-1050 m, very well coupled in the vertical direction. Also, internal waves of $3 \mathrm{~km}$ wavelength were observed in the depth range of 200$600 \mathrm{~m}$, and part of this scale also is well coupled in an oblique direction, from which we infer that internal waves of this scale in this part of the section have different propagating speed with depth. In the depth range of 600-1050 m, internal waves of $2.2 \mathrm{~km}$ wavelength, well coupled in the vertical direction, were also imaged. Finally, above $850 \mathrm{~m}$ water depth, there are internal waves of $6.5 \mathrm{~km}$ wavelength, separated by a clear interface at a depth of about $650 \mathrm{~m}$, which show a clear phase difference of about $90^{\circ}$ between the upper and lower parts; this is interpreted as indicating an oblique propagation of wave energy at this scale.

\section{Acknowledgments}

This research was supported co-financially by the National Major Fundamental Research and Development Project of China (no 2011CB403503) and China NSF (nos 91028002, 41076024). We would like to thank Professor Barry Ruddick for help during our seismic oceanographic cooperation. We are grateful to Dr Zhaohua Wu and Professor Norden Huang for their kind permission to use their modelling data. Our thanks are also given to the anonymous reviewers for very helpful suggestions and detailed comments.

\section{Appendix A. Scale mixing problems}

Scale mixing problems in 1D signals are often caused by intermittency (Wu and Huang 2009, Wu et al 2009). Not only the intermittency of the whole signal, but also intermittency at certain scales will lead to scale mixing. There are three main types of causes for scale mixing.
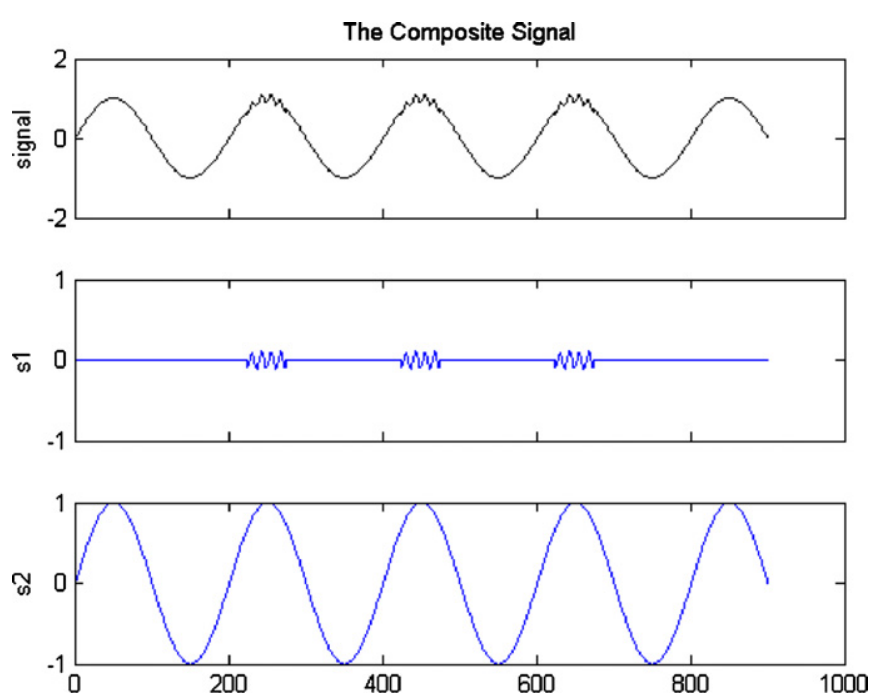

Figure A1. The signal used for testing the method is composed of the sum of two signals: s1 and s2 (after Huang et al (1999)). s1 is intermittent and its scale is comparatively small. $\mathrm{s} 2$ is a simple sinusoidal signal with a much larger scale. The vertical axis represents the amplitude of the signal and the horizontal axis represents the sampling points, without any particular physical meaning.

(1) Information from different scales exists in a single IMF.

(2) Information from the same scale is distributed between different levels of IMFs.

(3) When there are some relatively large (or small) values in a certain IMF, often a neighbouring IMF will have relatively small (or large) values at the corresponding position. This type of feature is artificial and has no physical meaning.

Tackling scale mixing problems is therefore extremely important because they will cause severe difficulties in the interpretation of the physical processes responsible for the generation of the data analysed.

Below, we show an example from Huang et al (1999) and $\mathrm{Wu}$ and Huang (2009), which illustrates how scale mixing problems occur when EMD is applied, and how they can be solved by EEMD. As mentioned before, scale mixing problems occur mainly because of intermittency in the signal. This makes the distribution of extrema non-uniform and therefore the range defined by the upper and lower envelopes, interpolated from the extrema, cannot cover the data completely and the mean of the upper and lower envelopes does not represent the exact trend of the signal (see, for example, figure A2 $(a)$, middle image). It should also be noted that, each time the sifting process subtracts the estimated trend from the signal, it will also likely subtract information concerning other scales from the original signal. Therefore, if the signal trend cannot be correctly defined, the final result, which will be an IMF, will of course have information from other scales and scale mixing problems will emerge. However, the added white noise will make the extrema be distributed uniformly throughout the whole signal when applying EEMD, and therefore the envelopes and trend can be defined more accurately, thereby reducing the scale mixing problems.

Figure A1 shows the signal used in this example, which is composed of the sum of two signals: s1 and s2. $\mathrm{s} 1$ is 

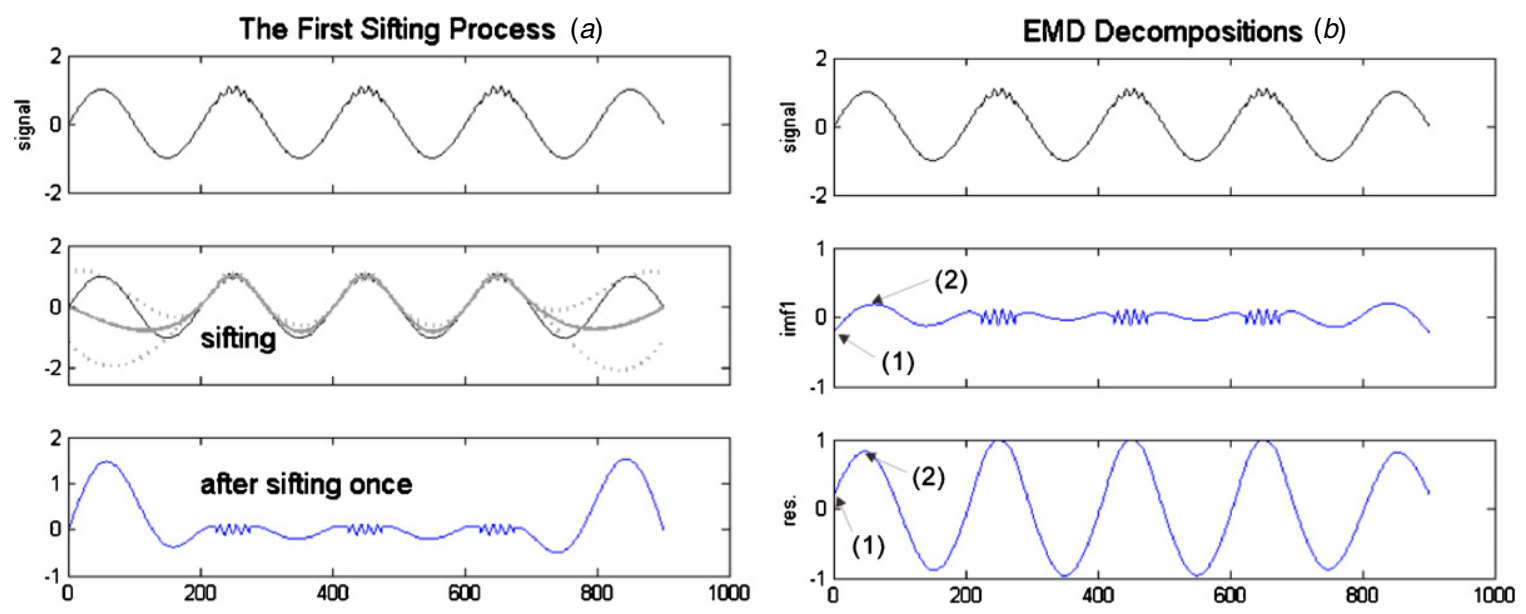

Figure A2. (a) The first sifting process and the corresponding result (recalculated after Wu and Huang (2009)). The first image is the original signal. The second image shows the process of sifting, in which the black solid line is the signal, the grey dotted lines are the envelopes (upper and lower) and the grey solid line is the mean of the envelopes. The third image is the result after sifting once. (b) The complete decompositions of the signal by EMD; imf1 is the first component, and res is the residual.

intermittent and its scale is comparatively small, whereas s 2 is a simple sinusoidal signal with a larger scale. According to the EMD theory, several sifting processes are necessary to extract an IMF. Figure A2 $(a)$ is the result after sifting once. We can see that there are many oscillations with a larger scale left in the result shown in the third row of figure A2(a); however only one sifting process was carried out. Figure A2(b) shows the final decomposition of the signal into imf1 and a residual (res). Again there are still some oscillations of small scale mixed with large scale in imf1, and this is the first type of scale mixing described above; oscillations of large scale also exist in both imf 1 and the residual — this is the second type of scale mixing; finally, at the location denoted as (1), because of the relatively small value of imf 1 , the corresponding location of the residual is relatively large, and at location (2) the opposite situation occurs - this is the third type of scale mixing.

Figure A3 shows the decomposition of the same signal shown in figure A2, using EEMD. Figure A3(a) shows the result when the ensemble number is set to 1 . Without stacking, the first three IMFs contain much noise besides the signal, but the purpose of avoiding scale mixing has been achieved for there are no oscillations of large scale in the first three IMFs. However, the result does not look so good without stacking. Figure $\mathrm{A} 3(b)$ is the result when the ensemble number is set to 100 . Now the result looks much better because the noise is nearly completely eliminated by stacking. In order to obtain a result with more physical meaning, the first three IMFs were combined into a new component $\mathrm{c} 1$, and the last two IMFs into another component $\mathrm{c} 2$, as shown in figure $\mathrm{A} 3(c)$. Figure A3 $(d)$ shows the differences between the original signal and the results from EEMD (diff1 is the result of s1 minus $\mathrm{c} 1$ and diff2 is the result of s2 minus c2). The locations marked by circles are those which earlier had relatively large differences, but that now look very small, confirming the excellent result obtained with EEMD.

\section{Appendix B. Sensitivity of EMD to perturbations}

Any digital signal processing method should be stable and robust, and therefore one does not expect very large changes in the result from just tiny oscillations in the input signal. However, the performance of EMD is not satisfactory in this respect (Wu and Huang 2009, Wu et al 2009). In the smooth area of a signal, if a tiny change is introduced at any point, the decompositions will show dramatic changes in a broad range centred at the perturbed point.

In the smooth area of the signal, there are a few extrema and therefore the interpolated envelopes can easily be significantly influenced by the 'outsider' perturbation. Because of such tiny perturbations, there will appear one or more extrema. Since the envelopes are interpolated from the extrema, the envelopes will change a lot around the perturbations, which will in turn cause the mean of envelopes to also change a lot, and this will affect the final decompositions. The affected area will be mainly between the two extrema closest to the perturbations, because when applying cubic splines, the area between two nodes (here referred to as extrema) is most affected by the two closest nodes; the other nodes will have less and less influence with increasing distance from the perturbation. The reason why EEMD can reduce sensitivity to perturbations is that the added white noise make the extrema distributed along the signal, and therefore the area affected by the local perturbation will be minimized.

Another example is shown in figure B1. Here, the original signal is now composed of the sum of two sinusoidal signals. Figure B1 (a) shows the resulting EMD decomposition. Because of the lack of adequate sampling rate and the influence of edge effects, the decompositions are not identical to the original sinusoidal signal, especially near the boundaries. Nevertheless, the overall pattern is fairly similar, and it was possible to successfully decompose the signal into two components. In figure B1(b), a small perturbation was added at point 100 in the horizontal axis (marked by the circle), and then the signal was again decomposed with EMD. From the 
(a)

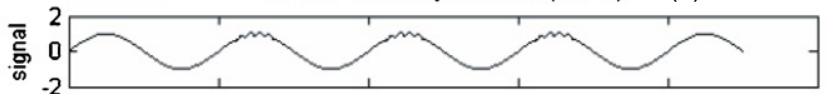

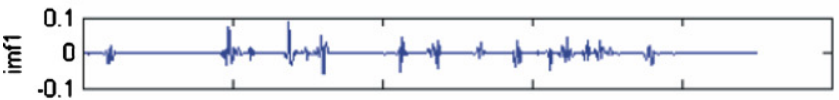

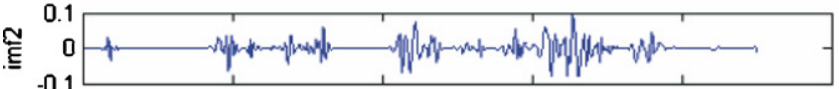

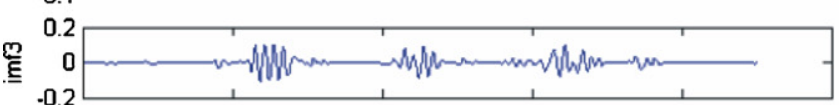
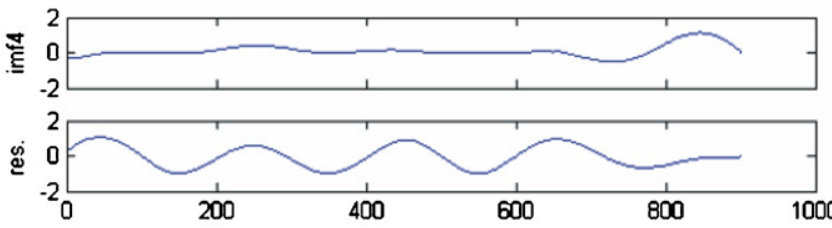

Combined EEMD Decompositions (c)
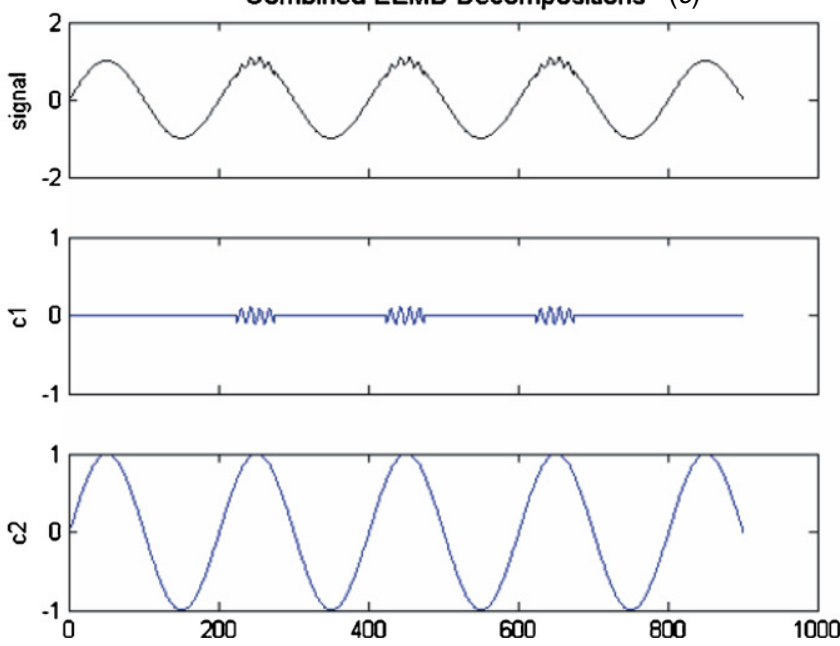

$(b)$
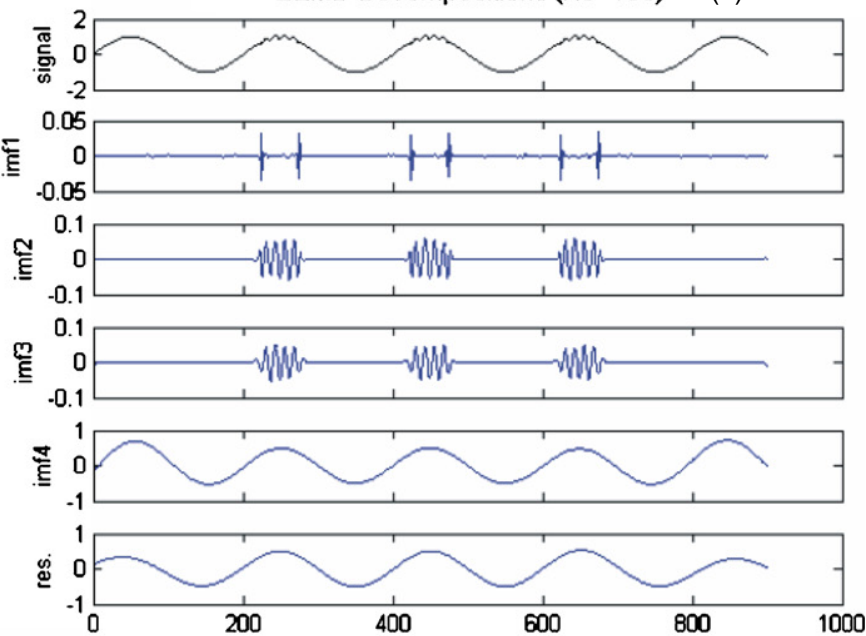

Difference

(d)
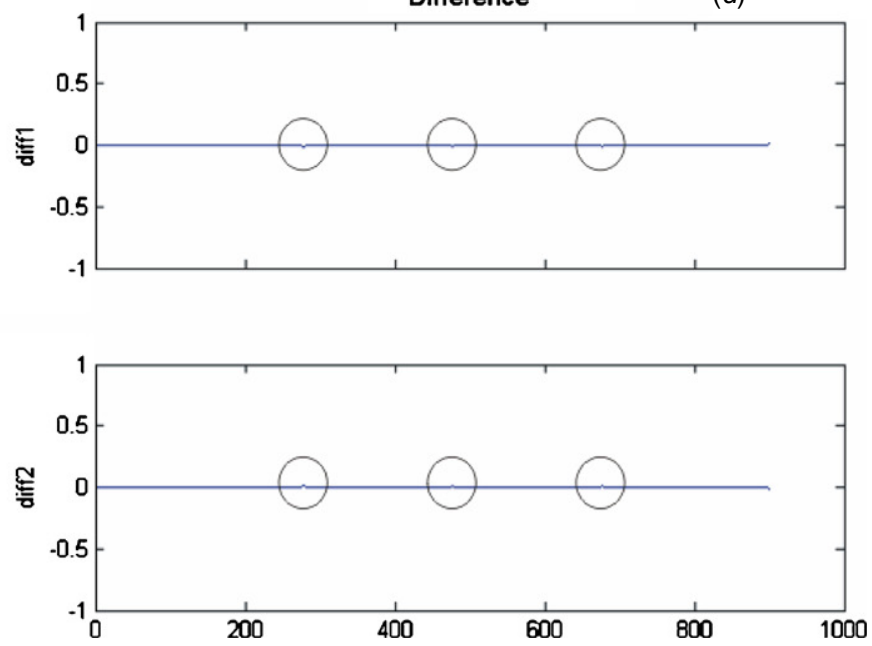

Figure A3. (a) EEMD decomposition of the synthetic signal shown in figure A1 for an ensemble number of 1 (NE $=1$ ). ( $b$ ) EEMD decomposition for $\mathrm{NE}=100 .(c) \mathrm{c} 1$ is the sum of $\mathrm{imf} 1$, imf 2 and imf 3 ; $\mathrm{c} 2$ is the sum of imf 4 and the residual. $(d)$ diff 1 is the difference between $\mathrm{c} 1$ and $\mathrm{s} 1$ (of figure A1); diff 2 is the difference between $\mathrm{c} 2$ and $\mathrm{s} 2$. The three circles mark the positions where some very small differences can be observed.
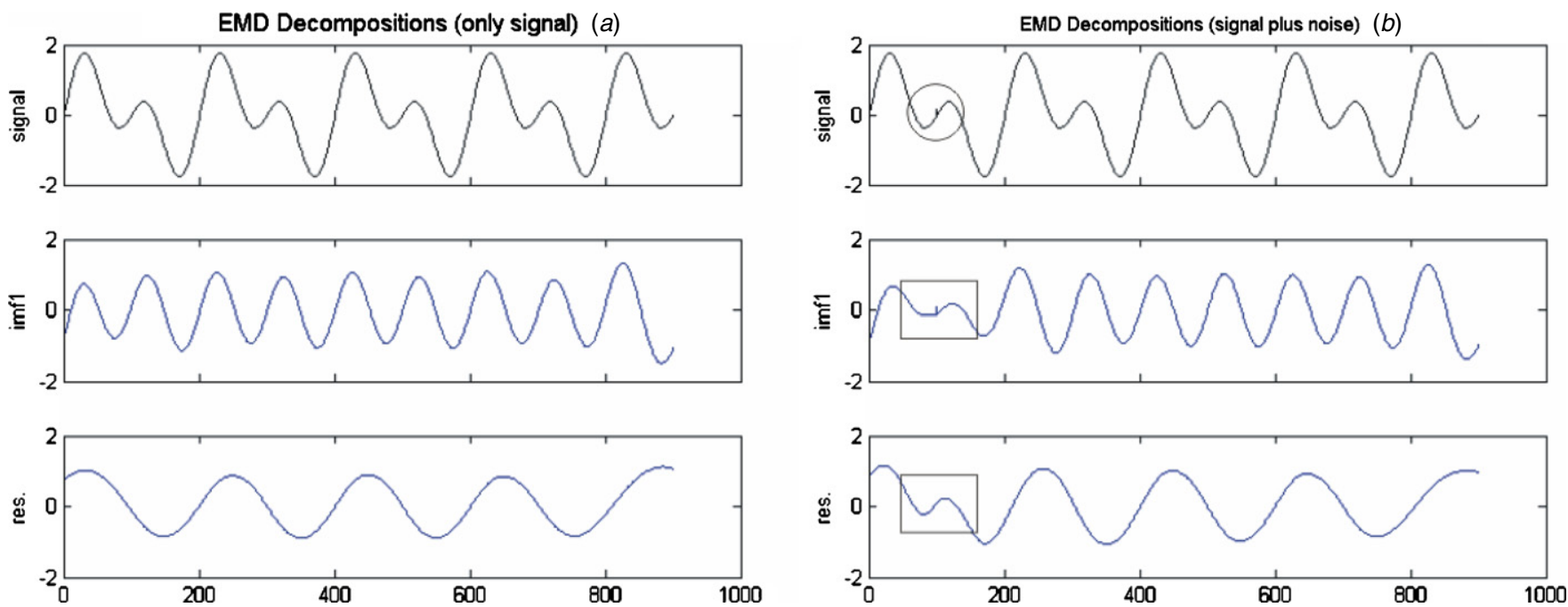

Figure B1. Synthetic signal composed of the sum of two sinusoidal curves. (a) The decompositions by EMD; $(b)$ the EMD decomposition when there is a small disturbance added at the position marked by the circle. Rectangles roughly mark the areas affected by the disturbance. 

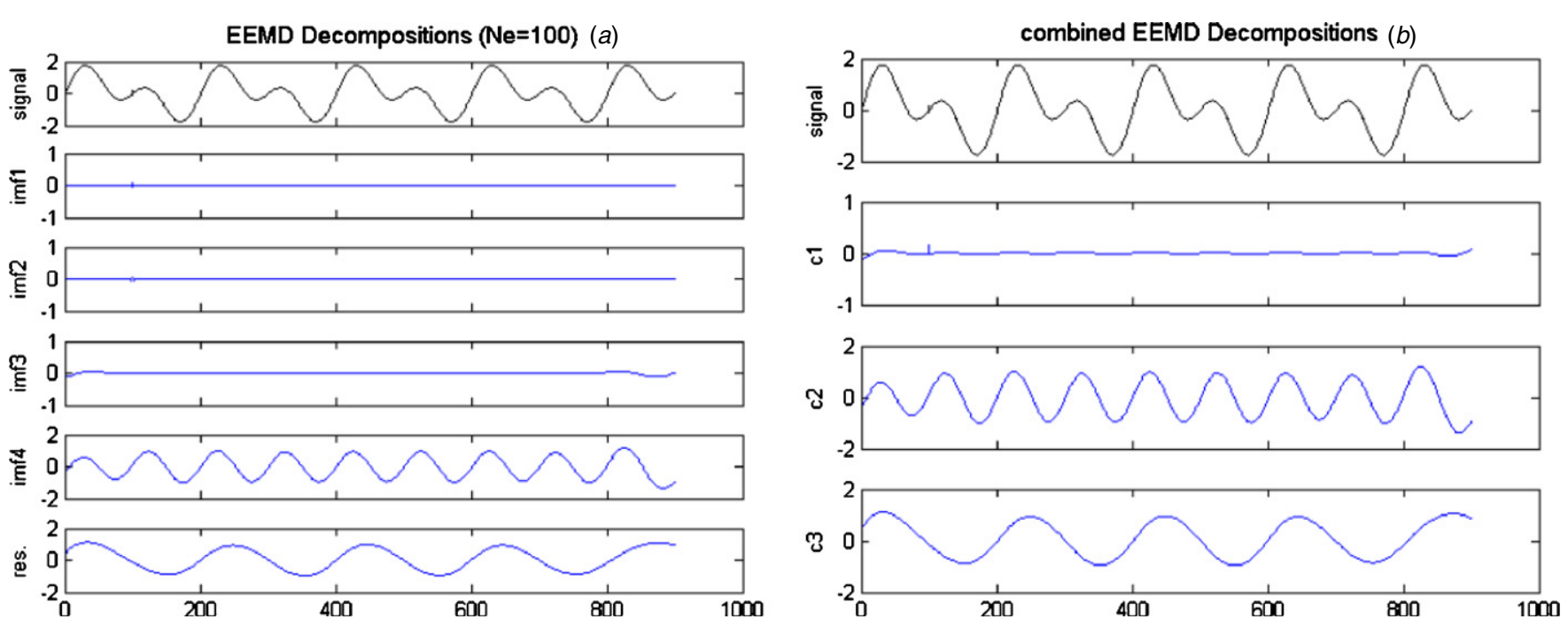

Figure B2. (a) EEMD decompositions of the signal in figure $\mathrm{B} 1(b)$, for $\mathrm{NE}=100 ;(b) \mathrm{c} 1$ is the sum of imf1, imf 2 and imf $3 ; \mathrm{c} 2$ is imf4; 3 is the residual.

next two images of figure B1 $(b)$ it can be observed that the results of decompositions change a lot within the area marked by the rectangle. This is what we would like to avoid, and is due to the sensitivity of EMD to perturbations, rather than related to any real physical meaning of the signal. Figure B2 shows the decompositions of the signal (same as shown in figure $\mathrm{B} 1(b))$ by EEMD with the ensemble number equal to 100 . Contrary to the results from EMD, not only has the stability of the decomposition process been guaranteed, but also the perturbation could be isolated. As shown in figure $\mathrm{B} 2(a)$, the perturbation was mainly contained in imf1 and imf2; imf3 contains little information of the original signal but has some edge effects. The main components of the signal are contained in imf 4 and the residual, respectively. Just as before, the first three IMFs were combined into a new component $\mathrm{c} 1$, imf 4 was treated as a new component $\mathrm{c} 2$, and the residual was treated as c3 (figure B2(b)).

\section{References}

Cai S Q and Gan Z J 2001 Progress in the study of the internal solition in the northern South China Sea Adv. Earth Sci. 16 215-9 (in Chinese)

Dong C Z, Song H B, Hao T Y, Chen L and Song Y 2009 Studying of oceanic internal wave spectra in the northeast South China Seas from seismic reflections Chin. J. Geophys. 52 2050-5 (in Chinese)

Fang X H and Du T 2005 Fundamental of Oceanic Waves and Internal Waves in the China Seas (Qingdao: China Ocean University Press) in Chinese

Flandrin P, Rilling G and Goncalves P 2004 Empirical mode decomposition as a filter bank IEEE Signal Process. Lett. $11112-4$

Garrett C and Munk W 1972 Space-time scales of internal waves Geophys. Astrophys. Fluid Dyn. 3 225-64

Garrett C and Munk W 1975 Space-time scales of internal waves: a progress report J. Geophys. Res. 80 291-7
Garrett C and Munk W 1979 Internal waves in the ocean Annu. Rev. Fluid Mech. 11 339-69

Holbrook W S and Fer I 2005 Ocean internal wave spectra inferred from seismic reflection transects Geophys. Res. Lett. 32 L15604

Holbrook W S, Paramo P, Pearse S and Schmitt R W 2003 Thermohaline fine structure in an oceanographic front from seismic reflection profiling Science 301 821-4

Huang N E, Shen Z and Long S R 1999 A new view of nonlinear water waves- the Hilbert spectrum Annu. Rev. Fluid Mech. 31 417-57

Huang N E, Shen Z, Long S R, Wu M C, Shin H H, Zheng Q A, Yen N C, Tung C C and Liu H H 1998 The empirical mode decomposition and the Hilbert spectrum for nonlinear and non-stationary time series analysis Proc. R. Soc. A 454 903-95

Jiang G R, Zhang J, Shi W L and Liu Q Y 2009 The Ocean Internal Waves and its Influence on Naval Battle (Beijing: China Meteorological Press) in Chinese

Liu A K, Chang Y S and Nai K L 1998 Evolution of nonlinear internal waves in the East and South China seas J. Geophys. Res. 103 7995-8008

Song H B, Bai Y, Dong C Z and Song Y 2010 A preliminary study of application of empirical mode decomposition method in understanding the features of internal waves in the northeastern South China Sea Chin. J. Geophys. 53 393-400 (in Chinese)

Song H B, Dong C Z, Chen L and Song Y 2008 Reflection seismic methods for studying physical oceanography: introduction of seismic oceanography Prog. Geophys. 23 1156-64 (in Chinese)

Song H B, Pinheiro L, Wang D X, Dong C Z, Song Y and Bai Y 2009 Seismic images of ocean meso scale eddies and internal waves Chin. J. Geophys. 52 2775-80 (in Chinese)

Wu Z H and Huang N E 2004 A study of the characteristics of white noise using the empirical mode decomposition method Proc. $R$. Soc. A 460 1597-611

Wu Z H and Huang N E 2009 Ensemble empirical mode decomposition: a noise-assisted data analysis method $A d v$. Adapt. Data Anal. 1 1-41

Wu Z H, Huang N E and Chen X Y 2009 The multi-dimensional ensemble empirical mode decomposition method Adv. Adapt. Data Anal. 1339-72 